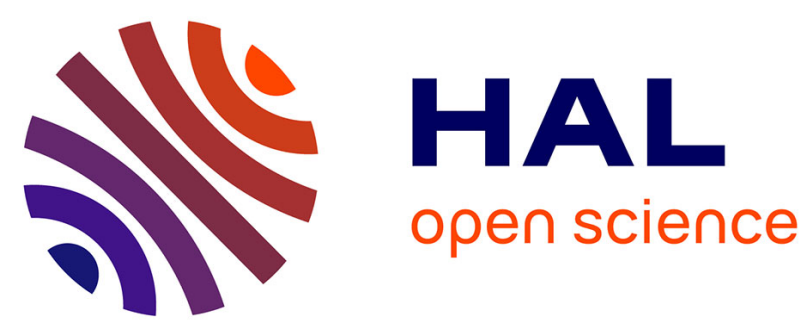

\title{
Resonant non-linear normal modes. Part I: analytical treatment for structural one-dimensional systems
}

\author{
Walter Lacarbonara, Giuseppe Rega, Ali Nayfeh
}

\section{To cite this version:}

Walter Lacarbonara, Giuseppe Rega, Ali Nayfeh. Resonant non-linear normal modes. Part I: analytical treatment for structural one-dimensional systems. International Journal of Non-Linear Mechanics, 2003, 38 (6), pp.851-872. 10.1016/S0020-7462(02)00033-1 . hal-01403851

\section{HAL Id: hal-01403851 \\ https://hal.science/hal-01403851}

Submitted on 28 Nov 2016

HAL is a multi-disciplinary open access archive for the deposit and dissemination of scientific research documents, whether they are published or not. The documents may come from teaching and research institutions in France or abroad, or from public or private research centers.
L'archive ouverte pluridisciplinaire HAL, est destinée au dépôt et à la diffusion de documents scientifiques de niveau recherche, publiés ou non, émanant des établissements d'enseignement et de recherche français ou étrangers, des laboratoires publics ou privés.

\section{(c)(1)}

Distributed under a Creative Commons Attribution| 4.0 International License 


\title{
Resonant non-linear normal modes. Part I: analytical treatment for structural one-dimensional systems
}

\author{
W. Lacarbonara ${ }^{a}$, G. Rega ${ }^{a, b}$, A.H. Nayfeh ${ }^{c}$ \\ ${ }^{a}$ Dipartimento di Ingegneria Strutturale e Geotecnica, Università di Roma La Sapienza, via Eudossiana, 18,00184 Roma, Italy \\ ${ }^{b}$ Dipartimento di Ingegneria Strutturale e Geotecnica, Università di Roma La Sapienza, via A. Gramsci, 53, 00197 Roma, Italy \\ ${ }^{\mathrm{c}}$ Department of Engineering Science and Mechanics, MC 0219, Virginia Polytechnic Institute and State University, \\ Blacksburg, VA 24061, USA
}

\begin{abstract}
Approximations of the resonant non-linear normal modes of a general class of weakly non-linear one-dimensional continuous systems with quadratic and cubic geometric non-linearities are constructed for the cases of two-to-one, one-to-one, and three-to-one internal resonances. Two analytical approaches are employed: the full-basis Galerkin discretization approach and the direct treatment, both based on use of the method of multiple scales as reduction technique. The procedures yield the uniform expansions of the displacement field and the normal forms governing the slow modulations of the amplitudes and phases of the modes. The non-linear interaction coefficients appearing in the normal forms are obtained in the form of infinite series with the discretization approach or as modal projections of second-order spatial functions with the direct approach. A systematic discussion on the existence and stability of coupled/uncoupled non-linear normal modes is presented. Closed-form conditions for non-linear orthogonality of the modes, in a global and local sense, are discussed. A mechanical interpretation of these conditions in terms of virtual works is also provided.
\end{abstract}

Keywords: Non-linear normal mode; Internal resonance; Non-linear orthogonality; Method of multiple scales; Virtual work

\section{Introduction}

Non-linear modal couplings due to internal resonances are possible in distributed-parameter systems depending on some geometrical and/or mechanical control parameters. Non-linear interactions between the modes are often also responsible for complex dynamical behaviors $[1,2]$.

On one hand, there is a theoretical interest in exploring the bifurcation behavior of the non-linear normal modes of continuous systems per se because this leads to a deeper understanding of the forced resonant dynamics when these modal interactions are activated. On the other hand, there is a practical interest in identifying a priori necessary and sufficient conditions for the actual activation (or non-activation) of non-linear modal interactions in continuous systems. In fact, when a system is designed to operate under dynamic loadings, often one of the designer's goals is to ascertain that the system will operate away from internal resonances leading to undesirable resonant multimode vibrations.

Starting with Rosenberg [3], the topic of nonlinear normal modes of conservative as well as 
non-conservative vibratory finite-degree-of-freedom and spatially continuous systems, with either weak or strong non-linearities, has been investigated quite extensively. Since then a variety of approximate analytical techniques has been proposed to construct the non-linear normal modes of discrete as well as continuous systems.

When treating spatially continuous systems, the approximate techniques include treatments of a discretized version with perturbation methods and direct reduction methods. With discretization methods, one assumes the solution as an expansion in terms of basis functions forming a complete set and then uses one of the variants of the method of weighted residuals (e.g., the Galerkin method) to obtain an infinite set of ordinary-differential equations usually truncated to a finite number of equations. Thereafter, the real-valued or complex-valued form of the invariant-manifold approach, the energy approach of King and Vakakis [4], or a perturbation method are used to treat the discretized equations.

On the other hand, direct analytical techniques [4-11] are available to construct the non-linear normal modes of continuous systems without a priori assumptions of the form of the solution. An exhaustive review of these techniques can be found in [2].

In a few previous works, while exploring internal resonances in some continuous systems, it was occasionally found that certain modes, although possessing frequencies in proper integer ratios with the potential for modal interaction activation, were not actually coupled. For example, investigating non-linear normal modes in clamped-clamped buckled beams [10], it was found that some modes could not interact at all, in spite of proper integer ratios between the associated frequencies, due to vanishing of the non-linear interaction coefficients in the normal forms.

Inspired by these results, we attempt to develop a general and systematic approach to determine a priori conditions for activation/non-activation of the modes under specific internal resonance conditions. The outcomes of these investigations are presented and discussed in this paper which is organized in two parts. The objective of Part I is twofold: (i) to study the existence and stability of coupled/uncoupled non-linear normal modes over variation of the internal resonance detuning in a general and systematic fashion using a set of partial-differential equations of motion and boundary conditions with general linear, quadratic, and cubic geometric operators; (ii) to determine closed-form conditions for the non-linear global and local orthogonality of the modes thereby extending the linear orthogonality concept applicable to self-adjoint systems entering the finite-amplitude vibration regime. In Part II [11], the general conditions are used for an in-depth analysis of non-linear orthogonality of the modes in various structural systems possessing symmetric and antisymmetric modes including buckled beams, shallow arches, and suspended cables.

The stated objectives are pursued, in the present paper, by constructing second-order uniform approximations of the coupled/uncoupled non-linear normal modes in the cases of two-to-one, three-to-one, and one-to-one internal resonances. The method of multiple scales [2] is applied to the infinite set of ordinary-differential equations obtained via the Galerkin method or directly to the partial-differential equations of motion and boundary conditions. In the first case, the full basis of the eigenfunctions of the associated linearized system is used as trial functions. The normal modes obtained with the full-basis Galerkin approach are the same as those obtained with direct application of the method of multiple scales to the equations of motion and boundary conditions as already shown in similar contexts [12-14]. However, the full-basis approach yields interesting modal representations of the coupled/uncoupled normal modes. These spectral representations, besides shedding light onto the structure of the non-linear modes, can be useful for modal convergence investigations (and reduced-order models selection). For a two-to-one internal resonance, we also discuss the effects of a higher-order approximation on the non-linear orthogonality and, in the case of activation, on the relative phase and amplitudes of the interacting modes.

Necessary and sufficient conditions for the local and global non-linear orthogonality of the modes are obtained in closed form. Two forms of non-linear orthogonality are illustrated: a local form and a global form. In the first case, uncoupling occurs in certain regions of the detuning-amplitude space. Therein, the modes cannot interact with periodic exchange of energy. In the second case, the orthogonality is global in the sense that it is independent on the detuning, the amplitudes, and the relative phases. We show that these 
global conditions can be interpreted as an extension of the linear orthogonality concept applicable to the modes of self-adjoint systems to their weak non-linear regime. Clearly, the obtained closed-form non-linear orthogonality conditions can save an enormous computational effort when it is needed to ascertain a priori whether orthogonality/non-orthogonality occurs in self-adjoint systems, the latter leading to modal interactions.

\section{A class of one-dimensional continuous systems}

Non-linear undamped unforced vibrations of a fairly general class of one-dimensional elastic continuous systems around their initially curved static non-linear configurations are governed, in non-dimensional form, by

$$
\ddot{\mathbf{u}}+\mathbf{L} \mathbf{u}=\mathbf{G}_{2}(\mathbf{u}, \mathbf{u})+\mathbf{G}_{3}(\mathbf{u}, \mathbf{u}, \mathbf{u})
$$

subject to the linear homogeneous boundary conditions

$\mathbf{B}_{i}(\mathbf{u})=\mathbf{0}, \quad i=1,2$

at both ends of the system. In (1), the overdot indicates differentiation with respect to the dimensionless time $t ; \mathbf{u}(x, t)$ denotes the dynamic displacements measured from the initially curved configuration and $x$ indicates the coordinate along the horizontal projection of the centerline of the system; $\mathbf{L}$ is a linear, homogeneous, self-adjoint, positive-definite integral-differential operator; $\mathbf{G}_{2}$ and $\mathbf{G}_{3}$ are quadratic and cubic geometric operators. The non-linear operators are non-commutative; i.e., $\mathbf{G}_{2}(\mathbf{v}, \mathbf{w}) \neq \mathbf{G}_{2}(\mathbf{w}, \mathbf{v})$. The operator notation here used is suitable to deal with general system-independent dynamics [14-17].

The eigenvalue problem governing the frequencies and associated mode shapes is

$$
\mathbf{L} \boldsymbol{\phi}-\omega^{2} \boldsymbol{\phi}=\mathbf{0}, \quad \mathbf{B}_{i}(\boldsymbol{\phi})=\mathbf{0} .
$$

By virtue of the self-adjoint nature of the linear unforced undamped problem, the mode shapes $\boldsymbol{\phi}_{m}(x)$-having in general in-plane and out-of-plane components - are mutually orthogonal and we assume they have been normalized such that $\int_{0}^{1} \boldsymbol{\phi}_{m}(x)^{\mathrm{T}} \boldsymbol{\phi}_{n}(x) \mathrm{d} x=\left\langle\boldsymbol{\phi}_{m} \boldsymbol{\phi}_{n}\right\rangle=\delta_{m n}$ and $\left\langle\boldsymbol{\phi}_{m}^{\mathrm{T}} \mathbf{L} \boldsymbol{\phi}_{n}\right\rangle=$ $\omega_{n}^{2} \delta_{m n}$, where $\delta_{m n}$ is the Kronecker delta and $\mathrm{T}$ indicates the transpose. It is worth observing that in many practical three-dimensional applications (e.g., suspended cables), the in-plane and out-of-plane eigenvalue problems are uncoupled. Therefore, there are two sets of eigenvalues and eigenfunctions, one associated with the in-plane problem and the other associated with the out-of-plane problem. In this case, it is easier to treat scalar differential equations rather than their vectorial counterparts. However, when we deal with coupled longitudinal and transverse vibrations of arches and suspended cables or with bending-torsional vibrations of beams, the kinematic configuration variables are coupled in the eigenvalue problem. In this case, the vector notation is an effective compact representation of the overally coupled system dynamics.

We use two approaches to the analytical construction of the non-linear normal modes: the discretization approach and the direct treatment, both based on the method of multiple scales as reduction technique. To avoid some drawbacks of the method of multiple scales applied to the equations of motion cast in second-order differential form (in time), we use the state-space formulation and cast the equations of motion in first-order differential form $[2,18]$ as follows:

$$
\begin{aligned}
& \dot{\mathbf{u}}-\mathbf{v}=0, \\
& \dot{\mathbf{v}}+\mathbf{L u}=\mathbf{G}_{2}(\mathbf{u}, \mathbf{u})+\mathbf{G}_{3}(\mathbf{u}, \mathbf{u}, \mathbf{u}) .
\end{aligned}
$$

In view of the analysis of the transverse dynamics of shallow systems which is tackled in Part II, longitudinal inertia can be neglected because the longitudinal modes are widely spaced from the transverse modes of vibration. This allows to use the classical condensation procedure for eliminating the longitudinal displacement component as a kinematic unknown. Consequently, to investigate modal interactions in the planar dynamics described by the transverse displacement component $u(x, t)$ only, we perform a full-basis discretization of (4) by letting $u(x, t)=$ $\sum_{k=1}^{\infty} q_{k}(t) \phi_{k}(x)$ and $v(x, t)=\sum_{k=1}^{\infty} z_{k}(t) \phi_{k}(x)$. Subsequently, we employ the Galerkin method leading to the following infinite set of non-linearly coupled 
ordinary-differential equations for planar interactions:

$$
\begin{aligned}
& \dot{q}_{k}-z_{k}=0, \\
& \dot{z}_{k}+\omega_{k}^{2} q_{k} \\
& =\sum_{i=1}^{\infty} \sum_{j=1}^{\infty} \Lambda_{k i j} q_{i} q_{j}+\sum_{i=1}^{\infty} \sum_{j=1}^{\infty} \sum_{h=1}^{\infty} \Gamma_{k i j h} q_{i} q_{j} q_{h}, \\
& \quad k=1,2, \ldots, \infty
\end{aligned}
$$

where

$$
\begin{array}{r}
\Lambda_{k i j}=\left\langle\phi_{k} G_{2}\left(\phi_{i}, \phi_{j}\right)\right\rangle \quad \text { and } \\
\Gamma_{k i j h}=\left\langle\phi_{k} G_{3}\left(\phi_{i}, \phi_{j}, \phi_{h}\right)\right\rangle .
\end{array}
$$

On the other hand, when studying modal interactions in the non-planar dynamics of cables described by two displacement components (in-plane and out-of-plane), the full-basis Galerkin discretization leads to two sets of coupled non-linear ordinary-differential equations, as documented in [11].

\section{Discretization approach}

In the present and following sections, we use the method of multiple scales [2] to construct uniform asymptotic expansions of the solutions of (4) and the associated boundary conditions in the case of small but finite (moderately large) dynamic displacements, and the expansions of the solutions of (5), when bimodal two-to-one, three-to-one, and one-to-one internal resonances may be activated. We use the discretization approach, first, and, successively, the direct treatment.

We assume that the generalized coordinates for the displacement and the velocity $q_{k}(t ; \varepsilon)$ and $z_{k}(t ; \varepsilon)$ can be expanded, respectively, as

$$
\begin{aligned}
q_{k}(t ; \varepsilon)= & \varepsilon q_{k 1}\left(T_{0}, T_{1}, T_{2}\right)+\varepsilon^{2} q_{k 2}\left(T_{0}, T_{1}, T_{2}\right) \\
& +\varepsilon^{3} q_{k 3}\left(T_{0}, T_{1}, T_{2}\right)+\cdots \\
z_{k}(t ; \varepsilon)= & \varepsilon z_{k 1}\left(T_{0}, T_{1}, T_{2}\right)+\varepsilon^{2} z_{k 2}\left(T_{0}, T_{1}, T_{2}\right) \\
& +\varepsilon^{3} z_{k 3}\left(T_{0}, T_{1}, T_{2}\right)+\cdots,
\end{aligned}
$$

where $q_{k}$ and $z_{k}$ are $O(\varepsilon)$ and $q_{k i}$ and $z_{k i}$ are $O(1)$, with $\varepsilon$ denoting a small non-dimensional parameter of the order of the amplitude of the solution and the symbol $O$ standing, as typical in asymptotic analyses, for "of the asymptotic order of" [19].
The time scales $T_{k}$ are defined as $T_{0}=t, T_{1}=\varepsilon t$, and $T_{2}=\varepsilon^{2} t$ and the first derivative with respect to time is defined as $\partial / \partial t=D_{0}+\varepsilon D_{1}+\varepsilon^{2} D_{2}+\cdots$, where $D_{n}=\partial / \partial T_{n}$.

Substituting (7), (8) and the time-derivative expansion into (5), and equating coefficients of equal powers of $\varepsilon$ yields

Order $\varepsilon$ :

$D_{0} q_{k 1}-z_{k 1}=0$,

$D_{0} z_{k 1}+\omega_{k}^{2} q_{k 1}=0$.

Order $\varepsilon^{2}$ :

$D_{0} q_{k 2}-z_{k 2}=-D_{1} q_{k 1}$,

$D_{0} z_{k 2}+\omega_{k}^{2} q_{k 2}=-D_{1} z_{k 1}+\sum_{j=1}^{\infty} \sum_{h=1}^{\infty} \Lambda_{k j h} q_{j 1} q_{h 1}$.

$\operatorname{Order} \varepsilon^{3}$ :

$D_{0} q_{k 3}-z_{k 3}=-D_{1} q_{k 2}-D_{2} q_{k 1}$,

$D_{0} z_{k 3}+\omega_{k}^{2} q_{k 3}$

$$
\begin{aligned}
= & -D_{1} z_{k 2}-D_{2} z_{k 1}+\sum_{j=1}^{\infty} \sum_{h=1}^{\infty} \Lambda_{k j h}\left(q_{j 1} q_{h 2}+q_{j 2} q_{h 1}\right) \\
& +\sum_{j=1}^{\infty} \sum_{h=1}^{\infty} \sum_{l=1}^{\infty} \Gamma_{k j h l} q_{j 1} q_{h 1} q_{l 1} .
\end{aligned}
$$

For bimodal interactions between the $m$ th and $n$th modes, the preliminary steps of the analysis are common to all of the internal resonances being investigated. Because the $m$ th and $n$th modes are the only modes involved in the internal resonance, we express the solutions of (9) and (10) as

$q_{k 1}=A_{k}\left(T_{1}, T_{2}\right) \mathrm{e}^{\mathrm{i} \omega_{k} T_{0}}\left(\delta_{k m}+\delta_{k n}\right)+\mathrm{cc}$,

$z_{k 1}=\mathrm{i} \omega_{k} A_{k}\left(T_{1}, T_{2}\right) \mathrm{e}^{\mathrm{i} \omega_{k} T_{0}}\left(\delta_{k m}+\delta_{k n}\right)+\mathrm{cc}$,

where $A_{k}$ denotes the complex-valued amplitude of the $k$ th mode and cc indicates the complex conjugate of the preceding terms.

Substituting (15) and (16) into (11) and (12) yields

$$
\begin{aligned}
& D_{0} q_{k 2}-z_{k 2}=-\left(D_{1} A_{k}\right) \mathrm{e}^{\mathrm{i} \omega_{k} T_{0}}\left(\delta_{k m}+\delta_{k n}\right)+\mathrm{cc}, \\
& D_{0} z_{k 2}+\omega_{k}^{2} q_{k 2} \\
&=-\mathrm{i} \omega_{k}\left(D_{1} A_{k}\right) \mathrm{e}^{\mathrm{i} \omega_{k} T_{0}}\left(\delta_{k m}+\delta_{k n}\right) \\
& \quad+\Lambda_{k m m}\left[A_{m}^{2} \mathrm{e}^{2 \mathrm{i} \omega_{m} T_{0}}+A_{m} \bar{A}_{m}\right]
\end{aligned}
$$




$$
\begin{aligned}
& +\Lambda_{k n n}\left[A_{n}^{2} \mathrm{e}^{2 \mathrm{i} \omega_{n} T_{0}}+A_{n} \bar{A}_{n}\right] \\
& +\left(\Lambda_{k m n}+\Lambda_{k n m}\right) A_{m} A_{n} \mathrm{e}^{\mathrm{i}\left(\omega_{m}+\omega_{n}\right) T_{0}} \\
& +\left(\Lambda_{k m n}+\Lambda_{k n m}\right) A_{n} \bar{A}_{m} \mathrm{e}^{\mathrm{i}\left(\omega_{n}-\omega_{m}\right) T_{0}}+c c .
\end{aligned}
$$

In the next sections, we treat separately the cases of two-to-one, three-to-one, and one-to-one internal resonances and we construct systematically their second-order expansions.

\subsection{Two-to-one internal resonances $\omega_{n} \approx 2 \omega_{m}$}

Non-linear resonant terms due to a two-to-one internal resonance are associated with the quadratic non-linearities. They appear at second order. Hence, a second-order expansion corresponds to a higher-order approximation of the investigated interaction. Our interest in seeking a higher-order approximation of the non-linear normal modes due to a two-to-one internal resonance is twofold. First, we aim at a preliminary estimation of the influence of higher-order effects, in general, on the expansion and, more specifically, on the non-linear modal orthogonality in such a relatively simple case. Furthermore, by doing so, we attempt to establish a systematic approach to construct second-order expansions of the non-linear normal modes under various internal resonance conditions.

The nearness of the internal resonance is expressed as $\omega_{n}=2 \omega_{m}+\varepsilon \sigma$, where $\sigma$ is a detuning parameter of order $O(1)$. We note that, in force of the internal resonance condition, resonant terms appear in (17) and (18) when $k=m$ or $n$. That is,

$$
\begin{aligned}
D_{0} q_{m 2}-z_{m 2}=- & \left(D_{1} A_{m}\right) \mathrm{e}^{\mathrm{i} \omega_{m} T_{0}}+\mathrm{cc} \\
D_{0} z_{m 2}+\omega_{m}^{2} q_{m 2}= & -\mathrm{i} \omega_{m}\left(D_{1} A_{m}\right) \mathrm{e}^{\mathrm{i} \omega_{m} T_{0}} \\
& +\left(\Lambda_{m m n}+\Lambda_{m n m}\right) A_{n} \bar{A}_{m} \mathrm{e}^{\mathrm{i} \omega_{m} T_{0}} \mathrm{e}^{\mathrm{i} \sigma T_{1}} \\
& +\mathrm{cc}+\mathrm{NST}
\end{aligned}
$$

and

$D_{0} q_{n 2}-z_{n 2}=-\left(D_{1} A_{n}\right) \mathrm{e}^{\mathrm{i} \omega_{n} T_{0}}+\mathrm{cc}$

$$
\begin{aligned}
D_{0} z_{n 2}+\omega_{n}^{2} q_{n 2}= & -\mathrm{i} \omega_{n}\left(D_{1} A_{n}\right) \mathrm{e}^{\mathrm{i} \omega_{n} T_{0}} \\
& +\Lambda_{n m m} A_{m}^{2} \mathrm{e}^{\mathrm{i} \omega_{n} T_{0}} \mathrm{e}^{-\mathrm{i} \sigma T_{1}} \\
& +\mathrm{cc}+\mathrm{NST},
\end{aligned}
$$

where NST denotes non-resonant terms.

Therefore, to render the problems solvable, we impose the orthogonality of the right-hand sides of the inhomogeneous equations (19) and (20) and those of (21) and (22) to every solution of the associated adjoint homogeneous problem. The solutions of the adjoint problems are $\left[\mathrm{i} \omega_{k}, 1\right] \exp \left(-\mathrm{i} \omega_{k} T_{0}\right), k=m, n$. Imposing the orthogonality yields

$2 \mathrm{i} \omega_{m} D_{1} A_{m}=\left(\Lambda_{m m n}+\Lambda_{m n m}\right) A_{n} \bar{A}_{m} \mathrm{e}^{\mathrm{i} \sigma T_{1}}$,

$2 \mathrm{i} \omega_{n} D_{1} A_{n}=\Lambda_{n m m} A_{m}^{2} \mathrm{e}^{-\mathrm{i} \sigma T_{1}}$.

Solving (23) and (24) for $D_{1} A_{m}$ and $D_{1} A_{n}$ and substituting the results into the second-order equations (17) and (18), we obtain

$$
\begin{aligned}
D_{0} q_{k 2} & -z_{k 2} \\
= & \frac{\mathrm{i}}{2 \omega_{m}}\left(\Lambda_{m m n}+\Lambda_{m n m}\right) A_{n} \bar{A}_{m} \mathrm{e}^{\mathrm{i} \omega_{m} T_{0}} \mathrm{e}^{\mathrm{i} \sigma T_{1}} \delta_{k m} \\
& +\frac{\mathrm{i}}{2 \omega_{n}} \Lambda_{n m m} A_{m}^{2} \mathrm{e}^{\mathrm{i} \omega_{n} T_{0}} \mathrm{e}^{-\mathrm{i} \sigma T_{1}} \delta_{k n}+\mathrm{cc}, \\
D_{0} z_{k 2} & +\omega_{k}^{2} q_{k 2} \\
= & -\frac{1}{2}\left(\Lambda_{m m n}+\Lambda_{m n m}\right) A_{n} \bar{A}_{m} \mathrm{e}^{\mathrm{i} \omega_{m} T_{0}} \mathrm{e}^{\mathrm{i} \sigma T_{1}} \delta_{k m} \\
& -\frac{1}{2} \Lambda_{n m m} A_{m}^{2} \mathrm{e}^{\mathrm{i} \omega_{n} T_{0}} \mathrm{e}^{-\mathrm{i} \sigma T_{1}} \delta_{k n} \\
& +\Lambda_{k m m}\left[A_{m}^{2} \mathrm{e}^{2 \mathrm{i} \omega_{m} T_{0}}+A_{m} \bar{A}_{m}\right] \\
& +\Lambda_{k n n}\left[A_{n}^{2} \mathrm{e}^{2 \mathrm{i} \omega_{n} T_{0}}+A_{n} \bar{A}_{n}\right] \\
& +\left(\Lambda_{k m n}+\Lambda_{k n m}\right) A_{m} A_{n} \mathrm{e}^{\mathrm{i}\left(\omega_{m}+\omega_{n}\right) T_{0}} \\
& +\left(\Lambda_{k m n}+\Lambda_{k n m}\right) A_{n} \bar{A}_{m} \mathrm{e}^{\mathrm{i}\left(\omega_{n}-\omega_{m}\right) T_{0}}+\mathrm{cc} .
\end{aligned}
$$

The solution of the inhomogeneous equations (25) and (26) is unique and straightforward when $k \neq m$ and $n$; on the contrary, when $k=m$ or $n$, the coupled ordinary-differential equations (25) and (26) exhibit resonant terms in their right-hand sides as 
already pointed out. However, because these equations have been rendered solvable, their solutions can be determined within an arbitrary constant. To determine the solutions of (25) and (26) when $k=m$ and $n$, we let

$q_{m 2}^{(R)}=Q_{m 2} A_{n} \bar{A}_{m} \mathrm{e}^{\mathrm{i} \omega_{m} T_{0}} \mathrm{e}^{\mathrm{i} \sigma T_{1}}$,

$z_{m 2}^{(R)}=Z_{m 2} A_{n} \bar{A}_{m} \mathrm{e}^{\mathrm{i} \omega_{m} T_{0}} \mathrm{e}^{\mathrm{i} \sigma T_{1}}$,

$q_{n 2}^{(R)}=Q_{n 2} A_{m}^{2} \mathrm{e}^{\mathrm{i} \omega_{n} T_{0}} \mathrm{e}^{-\mathrm{i} \sigma T_{1}}$,

$z_{n 2}^{(R)}=Z_{n 2} A_{m}^{2} \mathrm{e}^{\mathrm{i} \omega_{n} T_{0}} \mathrm{e}^{-\mathrm{i} \sigma T_{1}}$,

where the superscript $R$ indicates the resonant part of the second-order solution associated with the $m$ th and $n$th coordinates, respectively, and $Q_{j 2}$ and $Z_{j 2}$ are constants. Substituting the assumed forms of the solutions, (27) and (28), into (25) or (26) when $k=m$ and $n$, we obtain linear relationships between $Q_{j 2}$ and $Z_{j 2}$ in the form

$Z_{m 2}=\mathrm{i} \omega_{m} Q_{m 2}-\frac{\mathrm{i}}{2 \omega_{m}}\left(\Lambda_{m m n}+\Lambda_{m n m}\right)$,

$Z_{n 2}=\mathrm{i} \omega_{n} Q_{n 2}-\frac{\mathrm{i}}{2 \omega_{n}} \Lambda_{n m m}$.

To remove the indeterminacy, we require that the solutions $\left[Q_{j 2}, Z_{j 2}\right]$ be orthogonal to the adjoints $\left[\mathrm{i} \omega_{j}, 1\right]$, for $j=m$ and $n$. That is, $\left[Q_{j 2}, Z_{j 2}\right] \cdot\left[\mathrm{i} \omega_{j}, 1\right]^{\mathrm{T}}=0$ where (.) indicates the dot product. The result is

$Q_{m 2}=\frac{\Lambda_{m m n}+\Lambda_{m n m}}{4 \omega_{m}^{2}}$ and $Q_{n 2}=\frac{\Lambda_{n m m}}{4 \omega_{n}^{2}}$,

$Z_{j 2}=-\mathrm{i} \omega_{j} Q_{j 2}, \quad$ for $\quad j=m, n$.

Then, we can express the second-order solutions as ${ }^{1}$

$$
\begin{aligned}
q_{k 2}= & \frac{\Lambda_{m m n}+\Lambda_{m n m}}{4 \omega_{m}^{2}} A_{n} \bar{A}_{m} \mathrm{e}^{\mathrm{i}\left(\omega_{n}-\omega_{m}\right) T_{0}} \delta_{k m} \\
& +\frac{\Lambda_{n m m}}{4 \omega_{n}^{2}} A_{m}^{2} \mathrm{e}^{2 \mathrm{i} \omega_{m} T_{0}} \delta_{k n}
\end{aligned}
$$

\footnotetext{
${ }^{1}$ When the term $\left(1-\delta_{k j}\right)$ is zero in these expressions, it is tacitly intended that the corresponding multiplicative function must be removed (in spite of its vanishing denominator).
}

$$
\begin{aligned}
& +\frac{\Lambda_{k m m}}{\omega_{k}^{2}-4 \omega_{m}^{2}} A_{m}^{2} \mathrm{e}^{2 \mathrm{i} \omega_{m} T_{0}}\left(1-\delta_{k n}\right) \\
& +\frac{\Lambda_{k m n}+\Lambda_{k n m}}{\omega_{k}^{2}-\left(\omega_{n}-\omega_{m}\right)^{2}} A_{n} \bar{A}_{m} \\
& \times \mathrm{e}^{\mathrm{i}\left(\omega_{n}-\omega_{m}\right) T_{0}}\left(1-\delta_{k m}\right)+\frac{\Lambda_{k m m}}{\omega_{k}^{2}} A_{m} \bar{A}_{m} \\
& +\frac{\Lambda_{k n n}}{\omega_{k}^{2}-4 \omega_{n}^{2}} A_{n}^{2} \mathrm{e}^{2 \mathrm{i} \omega_{n} T_{0}} \\
& +\frac{\Lambda_{k n n}}{\omega_{k}^{2}} A_{n} \bar{A}_{n}+\frac{\Lambda_{k m n}+\Lambda_{k n m}}{\omega_{k}^{2}-\left(\omega_{m}+\omega_{n}\right)^{2}} \\
& \times A_{m} A_{n} \mathrm{e}^{\mathrm{i}\left(\omega_{m}+\omega_{n}\right) T_{0}}+\mathrm{cc}, \\
& z_{k 2}=-\mathrm{i} \frac{\Lambda_{m m n}+\Lambda_{m n m}}{4 \omega_{m}} A_{n} \bar{A}_{m} \mathrm{e}^{\mathrm{i}\left(\omega_{n}-\omega_{m}\right) T_{0}} \delta_{k m} \\
& -\mathrm{i} \frac{\Lambda_{n m m}}{4 \omega_{n}} A_{m}^{2} \mathrm{e}^{2 \mathrm{i} \omega_{m} T_{0}} \delta_{k n} \\
& +2 \mathrm{i} \omega_{m} \frac{\Lambda_{k m m}}{\omega_{k}^{2}-4 \omega_{m}^{2}} A_{m}^{2} \mathrm{e}^{2 \mathrm{i} \omega_{m} T_{0}}\left(1-\delta_{k n}\right) \\
& +2 \mathrm{i} \omega_{n} \frac{\Lambda_{k n n}}{\omega_{k}^{2}-4 \omega_{n}^{2}} A_{n}^{2} \mathrm{e}^{2 \mathrm{i} \omega_{n} T_{0}} \\
& +\mathrm{i}\left(\omega_{n}-\omega_{m}\right) \frac{\Lambda_{k m n}+\Lambda_{k n m}}{\omega_{k}^{2}-\left(\omega_{n}-\omega_{m}\right)^{2}} \\
& \times A_{n} \bar{A}_{m} \mathrm{e}^{\mathrm{i}\left(\omega_{n}-\omega_{m}\right) T_{0}}\left(1-\delta_{k m}\right) \\
& +\mathrm{i}\left(\omega_{m}+\omega_{n}\right) \frac{\Lambda_{k m n}+\Lambda_{k n m}}{\omega_{k}^{2}-\left(\omega_{m}+\omega_{n}\right)^{2}} \\
& \times A_{m} A_{n} \mathrm{e}^{\mathrm{i}\left(\omega_{m}+\omega_{n}\right) T_{0}}+\mathrm{cc} .
\end{aligned}
$$

Substituting the second-order solutions, (32) and (33), into the third-order problem, (13) and (14), and imposing again solvability conditions yields the modulation equations governing the dependence of the complex-valued amplitudes $A_{m}$ and $A_{n}$ on the scale $T_{2}$ in the form

$2 \mathrm{i} \omega_{m} D_{2} A_{m}=K_{m m} A_{m}^{2} \bar{A}_{m}+K_{m n} A_{m} A_{n} \bar{A}_{n}$

$2 \mathrm{i} \omega_{n} D_{2} A_{n}=K_{n n} A_{n}^{2} \bar{A}_{n}+K_{n m} A_{n} A_{m} \bar{A}_{m}$, 
where the coefficients $K_{h h}$ and $K_{m n}$ are given by the following infinite series:

$$
\begin{aligned}
K_{m m}= & \sum_{j=1, j \neq n}^{\infty}\left[\left(\Lambda_{m m j}+\Lambda_{m j m}\right)\right. \\
& \left.\times\left(\frac{2 \Lambda_{j m m}}{\omega_{j}^{2}}+\frac{\Lambda_{j m m}}{\omega_{j}^{2}-4 \omega_{m}^{2}}\right)\right] \\
& +\frac{9}{4 \omega_{n}^{2}} \Lambda_{n m m}\left(\Lambda_{m m n}+\Lambda_{m n m}\right)+3 \Gamma_{m m m m}, \\
K_{n n}= & \sum_{j=1}^{\infty}\left[\left(\Lambda_{n n j}+\Lambda_{n j n}\right)\left(\frac{2 \Lambda_{j n n}}{\omega_{j}^{2}}+\frac{\Lambda_{j n n}}{\omega_{j}^{2}-4 \omega_{n}^{2}}\right)\right] \\
& +3 \Gamma_{n n n n}, \\
K_{m n}= & \sum_{j=1, j \neq m}^{\infty}\left[\left(\Lambda_{m j n}+\Lambda_{m n j}\right)\left(\Lambda_{j m n}+\Lambda_{j n m}\right)\right. \\
& \times\left(\frac{1}{\omega_{j}^{2}-9 \omega_{m}^{2}}+\frac{1}{\omega_{j}^{2}-\omega_{m}^{2}}\right) \\
& \left.+\frac{2 \Lambda_{j n n}}{\omega_{j}^{2}}\left(\Lambda_{m m j}+\Lambda_{m j m}\right)\right] \\
& +\frac{\left(\Lambda_{m m n}+\Lambda_{m n m}\right)^{2}}{8 \omega_{m}^{2}}+\frac{4}{\omega_{m}^{2}}\left(\Lambda_{m n n} \Lambda_{m m m}\right) \\
& +2\left(\Gamma_{m n n m}+\Gamma_{m n m n}+\Gamma_{m m n n}\right) .
\end{aligned}
$$

Using the method of reconstitution [2], we can express the derivative of $A$ with respect to time $t$ as $\dot{A}=\varepsilon D_{1} A+\varepsilon^{2} D_{2} A+\cdots$. Therefore, substituting (23), (24), (34), and (35) into this equation, we obtain the modulation equations up to third order as

$$
\begin{aligned}
2 \mathrm{i} \omega_{m} \dot{A}_{m}= & \left(\Lambda_{m m n}+\Lambda_{m n m}\right) A_{n} \bar{A}_{m} \mathrm{e}^{\mathrm{i} \sigma t}+K_{m m} A_{m}^{2} \bar{A}_{m} \\
& +K_{m n} A_{m} A_{n} \bar{A}_{n},
\end{aligned}
$$

$$
\begin{aligned}
2 \mathrm{i} \omega_{n} \dot{A}_{n}= & \Lambda_{n m m} A_{m}^{2} \mathrm{e}^{-\mathrm{i} \sigma t}+K_{n n} A_{n}^{2} \bar{A}_{n} \\
& +K_{n m} A_{n} A_{m} \bar{A}_{m},
\end{aligned}
$$

where $\varepsilon$ was, for convenience, set equal to unity (this is admissible for the bookkeeping function of $\varepsilon$ ).
By virtue of the conservative nature of the problem, (39) and (40) must be derivable from the Lagrangian

$L=T-\left(V^{(N)}+V^{(\sigma)}\right)$,

where

$T=\mathrm{i} \omega_{n}\left(A_{n} \dot{\bar{A}}_{n}-\bar{A}_{n} \dot{A}_{n}\right)+\mathrm{i} \omega_{m}\left(A_{m} \dot{\bar{A}}_{m}-\bar{A}_{m} \dot{A}_{m}\right)$

is the system kinetic energy associated with the interaction;

$$
\begin{aligned}
V^{(N)}= & -\frac{1}{2}\left(K_{n n} A_{n}^{2} \bar{A}_{n}^{2}+K_{m m} A_{m}^{2} \bar{A}_{m}^{2}\right. \\
& \left.+2 K_{m n} A_{n} \bar{A}_{n} A_{m} \bar{A}_{m}\right)
\end{aligned}
$$

is part of the Lagrangian associated with the resonance-independent non-linear elastic potential energy (the superscript $N$ indicates the non-resonant part of the Lagrangian); and

$V^{(\sigma)}=-\frac{1}{2}\left(S A_{n} \bar{A}_{m}^{2} \mathrm{e}^{\mathrm{i} \sigma t}+\mathrm{cc}\right)$

is part of the Lagrangian associated with the resonance detuning-dependent elastic potential energy.

Consequently, imposing that (39) and (40) satisfy the Euler-Lagrange equations [20] based on the Lagrangian (41) yields

$S=S_{m}=2 S_{n}$,

$K_{m n}=K_{n m}$,

where $S_{m}=\Lambda_{m m n}+\Lambda_{m n m}$ and $S_{n}=\Lambda_{n m m}$.

Letting $A_{k}(t)=(1 / 2) a_{k}(t) \exp (\mathrm{i} \beta(t))$, we can express the first- and second-order generalized coordinates as

$$
\begin{aligned}
q_{k 1}= & a_{k} \cos \left(\omega_{k} t+\beta_{k}\right)\left(\delta_{k m}+\delta_{k n}\right), \\
q_{k 2}= & \frac{1}{2} a_{m}^{2} \frac{\Lambda_{n m m}}{4 \omega_{n}^{2}} \cos 2\left(\omega_{m} t+\beta_{m}\right) \delta_{k n} \\
& +\frac{1}{2} \frac{\left(\Lambda_{m m n}+\Lambda_{m n m}\right)}{4 \omega_{m}^{2}} a_{m} a_{n} \\
& \times \cos \left(\left(\omega_{n}-\omega_{m}\right) t+\beta_{n}-\beta_{m}\right) \delta_{k m} \\
& +\frac{1}{2} a_{m}^{2} \frac{\Lambda_{k m m}}{\omega_{k}^{2}}+\frac{1}{2} a_{n}^{2} \\
& \times\left[\frac{\Lambda_{k n n}}{\omega_{k}^{2}-4 \omega_{n}^{2}} \cos 2\left(\omega_{n} t+\beta_{n}\right)+\frac{\Lambda_{k n n}}{\omega_{k}^{2}}\right]
\end{aligned}
$$




$$
\begin{aligned}
& +\frac{1}{2} a_{m} a_{n} \frac{\left(\Lambda_{k m n}+\Lambda_{k n m}\right)}{\omega_{k}^{2}-\left(\omega_{m}+\omega_{n}\right)^{2}} \\
& \times \cos \left(\left(\omega_{m}+\omega_{n}\right) t+\beta_{m}+\beta_{n}\right) \\
& +\frac{1}{2} a_{m}^{2} \frac{\Lambda_{k m m}}{\omega_{k}^{2}-4 \omega_{m}^{2}} \cos 2\left(\omega_{m} t+\beta_{m}\right)\left(1-\delta_{k n}\right) \\
& +\frac{1}{2} a_{m} a_{n} \frac{\left(\Lambda_{k m n}+\Lambda_{k n m}\right)}{\omega_{k}^{2}-\left(\omega_{n}-\omega_{m}\right)^{2}} \\
& \times \cos \left(\left(\omega_{n}-\omega_{m}\right) t+\beta_{n}-\beta_{m}\right)\left(1-\delta_{k m}\right) .
\end{aligned}
$$

Using the assumed full-basis modal expansion for the displacement $u(x, t)=\sum_{k=1}^{\infty} q_{k}(t) \phi_{k}(x)$, the latter can be expressed, up to second order, as

$$
\begin{aligned}
u(x, t)= & a_{n} \cos \left(\omega_{n} t+\beta_{n}\right) \phi_{n}(x) \\
& +a_{m} \cos \left(\omega_{m} t+\beta_{m}\right) \phi_{m}(x) \\
& +\frac{1}{2}\left\{a_{n}^{2}\left[\cos 2\left(\omega_{n} t+\beta_{n}\right) \psi_{n n}(x)+\kappa_{n n}(x)\right]\right. \\
& +a_{m}^{2}\left[\cos 2\left(\omega_{m} t+\beta_{m}\right) \psi_{m m}(x)+\kappa_{m m}(x)\right] \\
& +a_{n} a_{m}\left[\cos \left(\left(\omega_{n}+\omega_{m}\right) t+\beta_{n}+\beta_{m}\right) \psi_{m n}(x)\right. \\
& \left.\left.+\cos \left(\left(\omega_{n}-\omega_{m}\right) t+\beta_{n}-\beta_{m}\right) \kappa_{m n}(x)\right]\right\},(49)
\end{aligned}
$$

where $\varepsilon$ was reabsorbed in the amplitude expressions and the second-order shape functions are given by

$$
\begin{aligned}
\psi_{m m}(x)= & \sum_{k=1, k \neq n}^{\infty} \frac{\Lambda_{k m m}}{\omega_{k}^{2}-4 \omega_{m}^{2}} \phi_{k}(x) \\
& +\frac{\Lambda_{n m m}}{4 \omega_{n}^{2}} \phi_{n}(x), \\
\kappa_{m n}(x)= & \sum_{k=1, k \neq m}^{\infty} \frac{\Lambda_{k m n}+\Lambda_{k n m}}{\omega_{k}^{2}-\left(\omega_{n}-\omega_{m}\right)^{2}} \phi_{k}(x) \\
& +\frac{\Lambda_{m m n}+\Lambda_{m n m}}{4 \omega_{m}^{2}} \phi_{m}(x), \\
\psi_{n n}(x)= & \sum_{k=1}^{\infty} \frac{\Lambda_{k n n}}{\omega_{k}^{2}-4 \omega_{n}^{2}} \phi_{k}(x), \\
\psi_{m n}(x)= & \sum_{k=1}^{\infty} \frac{\Lambda_{k m n}+\Lambda_{k n m}}{\omega_{k}^{2}-\left(\omega_{m}+\omega_{n}\right)^{2}} \phi_{k}(x),
\end{aligned}
$$

$$
\begin{aligned}
& \kappa_{m m}(x)=\sum_{k=1}^{\infty} \frac{\Lambda_{k m m}}{\omega_{k}^{2}} \phi_{k}(x), \\
& \kappa_{n n}(x)=\sum_{k=1}^{\infty} \frac{\Lambda_{k n n}}{\omega_{k}^{2}} \phi_{k}(x) .
\end{aligned}
$$

In (49), the amplitudes and phases are governed by (39) and (40). We also observe that the functions $\kappa_{m m}$ and $\kappa_{n n}$ are associated with zero frequency; hence, they govern the new equilibrium configuration produced by the quadratic non-linearities around which the system periodically oscillates. Clearly, the displacement field (49) depends, in principle, on all of the eigenmodes, directly, through (50)(53) denoting second-order spatial corrections and, indirectly, through the amplitudes and phases which are solutions of the modulation equations governed by the coefficients $K_{i j}$ and $S$. In these coefficients, the quadratic non-linearities produce contributions from all of the modes as also documented in [14]. On the contrary, the effects of the cubic non-linearities, for second-order expansions, are accounted for solely by the mode shapes involved in the resonance.

It is also clear that high-frequency modal contributions are less significant either in the spatial functions or in the amplitudes and phases through the interaction coefficients, because the associated frequencies appear (in the denominators of the shape functions $\psi_{i j}\left(\kappa_{i j}\right)$ or in those of the coefficients) squared or as the difference of the square of the frequency and the square of the dominant frequencies. The modal convergence of the non-linear normal modes depends on the rate of convergence of the series expressing the interaction coefficients.

\subsection{Three-to-one internal resonances $\omega_{n} \approx 3 \omega_{m}$}

The analysis up to (18) holds for a three-to-one as well as a one-to-one internal resonance. The difference is that these internal resonances are associated with third-order effects; hence, the solutions do not depend on the time scale $T_{1}$. Consequently, the solutions of (5) are assumed in the form of (7) and (8) neglecting the dependence on $T_{1}$. 
We can express the solutions of (17) and (18) with $D_{1} \equiv 0$ as

$$
\begin{aligned}
q_{k 2}\left(T_{0}, T_{2}\right) & \\
= & \frac{\Lambda_{k m m}}{\omega_{k}^{2}-4 \omega_{m}^{2}} A_{m}^{2} \mathrm{e}^{2 \mathrm{i} \omega_{m} T_{0}}+\frac{\Lambda_{k m m}}{\omega_{k}^{2}} A_{m} \bar{A}_{m} \\
& +\frac{\Lambda_{k n n}}{\omega_{k}^{2}-4 \omega_{n}^{2}} A_{n}^{2} \mathrm{e}^{2 \mathrm{i} \omega_{n} T_{0}}+\frac{\Lambda_{k n n}}{\omega_{k}^{2}} A_{n} \bar{A}_{n} \\
& +\frac{\Lambda_{k m n}+\Lambda_{k n m}}{\omega_{k}^{2}-\left(\omega_{m}+\omega_{n}\right)^{2}} A_{m} A_{n} \mathrm{e}^{\mathrm{i}\left(\omega_{m}+\omega_{n}\right) T_{0}} \\
& +\frac{\Lambda_{k m n}+\Lambda_{k n m}}{\omega_{k}^{2}-\left(\omega_{n}-\omega_{m}\right)^{2}} A_{n} \bar{A}_{m} \mathrm{e}^{\mathrm{i}\left(\omega_{n}-\omega_{m}\right) T_{0}}+\mathrm{cc},
\end{aligned}
$$

$z_{k 2}=D_{0} q_{k 2}$.

Substituting (54) and (55) into (13) and (14) yields resonant terms in the $m$ th and $n$th third-order equations depending on the type of internal resonance. Expressing the nearness of the three-to-one internal resonance as $\omega_{n}=3 \omega_{m}+\varepsilon^{2} \sigma$ and imposing the solvability conditions yields the following modulation equations:

$$
\begin{aligned}
2 \mathrm{i} \omega_{m} D_{2} A_{m}= & K_{m m} A_{m}^{2} \bar{A}_{m}+K_{m n} A_{m} A_{n} \bar{A}_{n} \\
& +\Lambda_{m} A_{n} \bar{A}_{m}^{2} \mathrm{e}^{\mathrm{i} \sigma T_{2}}, \\
2 \mathrm{i} \omega_{n} D_{2} A_{n}= & K_{n n} A_{n}^{2} \bar{A}_{n}+K_{n m} A_{n} A_{m} \bar{A}_{m} \\
& +\Lambda_{n} A_{m}^{3} \mathrm{e}^{-\mathrm{i} \sigma T_{2}} .
\end{aligned}
$$

The coefficients $K_{h h}, K_{m n}$, and $\Lambda_{n}$ are obtained as

$$
\begin{aligned}
K_{h h}= & \sum_{j=1}^{\infty}\left[\left(\Lambda_{h h j}+\Lambda_{h j h}\right)\left(\frac{2 \Lambda_{j h h}}{\omega_{j}^{2}}+\frac{\Lambda_{j h h}}{\omega_{j}^{2}-4 \omega_{h}^{2}}\right)\right] \\
& +3 \Gamma_{h h h h, \quad h=m, n,} \\
K_{m n}= & \sum_{j=1}^{\infty}\left[\left(\Lambda_{m m j}+\Lambda_{m j m}\right) \frac{2 \Lambda_{j n n}}{\omega_{j}^{2}}\right. \\
& +\left(\Lambda_{m n j}+\Lambda_{m j n}\right)\left(\Lambda_{j m n}+\Lambda_{j n m}\right) \\
& \left.\times\left(\frac{1}{\omega_{j}^{2}-\left(\omega_{n}+\omega_{m}\right)^{2}}+\frac{1}{\omega_{j}^{2}-\left(\omega_{n}-\omega_{m}\right)^{2}}\right)\right] \\
& +2\left(\Gamma_{m n n m}+\Gamma_{m n m n}+\Gamma_{m m n n}\right),
\end{aligned}
$$

$$
\Lambda_{n}=\sum_{j=1}^{\infty}\left[\left(\Lambda_{n m j}+\Lambda_{n j m}\right) \frac{\Lambda_{j m m}}{\omega_{j}^{2}-4 \omega_{m}^{2}}\right]+\Gamma_{n m m m} .
$$

Again, exploiting the conservative nature of the problem, we impose that (56) and (57) be derivable from the Lagrangian expressed by (41)-(43) with the coefficients given by (58) and (59)

$$
V^{(\sigma)}=-\frac{\Lambda}{3}\left(A_{n} \bar{A}_{m}^{3} \mathrm{e}^{\mathrm{i} \sigma t}+\mathrm{cc}\right) .
$$

In addition to the resonance-independent symmetry condition (46) (i.e., $K_{m n}=K_{n m}$ ), we obtain the following resonance-dependent condition:

$\Lambda=\Lambda_{m}=3 \Lambda_{n}$.

The displacement field is expressed again by (49) with $\psi_{n n}, \psi_{m n}, \kappa_{m m}$, and $\kappa_{n n}$ given by (52) and (53), whereas $\psi_{m m}$ and $\kappa_{m n}$ are replaced, respectively, with

$$
\begin{aligned}
& \psi_{m m}(x)=\sum_{k=1}^{\infty} \frac{\Lambda_{k m m}}{\omega_{k}^{2}-4 \omega_{m}^{2}} \phi_{k}(x), \\
& \kappa_{m n}(x)=\sum_{k=1}^{\infty} \frac{\Lambda_{k m n}+\Lambda_{k n m}}{\omega_{k}^{2}-\left(\omega_{n}-\omega_{m}\right)^{2}} \phi_{k}(x) .
\end{aligned}
$$

\subsection{One-to-one internal resonances $\omega_{n} \approx \omega_{m}$}

One-to-one internal resonances may occur in two cases: at crossover points (where the frequencies of two or more modes coalesce by transversal intersection over variation of a system parameter [21] or at veering points (where the frequencies of two or more modes coalesce by tangential intersection [22]). For example, in the case of suspended cables with supports at different levels, it was found [23] that the eigenfunctions are a mixture of symmetric and antisymmetric components. In this case, the curve veering phenomenon occurs between the frequencies of the lowest two modes upon variation of a modified cable parameter accounting for its elasto-geometric properties including the inclined geometry.

For a one-to-one internal resonance, we express its detuning as $\omega_{n}=\omega_{m}+\varepsilon^{2} \sigma$. For a one-to-one internal resonance at a crossover point, the detuning parameter can be either positive or negative whereas for a one-to-one internal resonance at a veering point, the detuning parameter does not change sign. 
Nevertheless, the theoretical analysis performed here holds also for the veering case.

Substituting (15) and (16), (54), and (55) into (13) and (14), and imposing that the inhomogeneous equations be solvable leads to the following modulation equations:

$$
\begin{aligned}
2 \mathrm{i} \omega_{m} D_{2} A_{m}= & K_{m m} A_{m}^{2} \bar{A}_{m}+K_{m n} A_{m} A_{n} \bar{A}_{n}+R_{5} A_{n}^{2} \bar{A}_{n} \mathrm{e}^{\mathrm{i} \sigma T_{2}} \\
& +R_{6} A_{n} A_{m} \bar{A}_{m} \mathrm{e}^{\mathrm{i} \sigma T_{2}}+R_{7} A_{m}^{2} \bar{A}_{n} \mathrm{e}^{-\mathrm{i} \sigma T_{2}} \\
& +R_{8} A_{n}^{2} \bar{A}_{m} \mathrm{e}^{2 \mathrm{i} \sigma T_{2}} \\
2 \mathrm{i} \omega_{n} D_{2} A_{n}= & K_{n n} A_{n}^{2} \bar{A}_{n}+K_{n m} A_{n} A_{m} \bar{A}_{m}+R_{1} A_{m}^{2} \bar{A}_{m} \mathrm{e}^{-\mathrm{i} \sigma T_{2}} \\
& +R_{2} A_{m} A_{n} \bar{A}_{n} \mathrm{e}^{-\mathrm{i} \sigma T_{2}}+R_{3} A_{m}^{2} \bar{A}_{n} \mathrm{e}^{-2 \mathrm{i} \sigma T_{2}} \\
& +R_{4} A_{n}^{2} \bar{A}_{m} \mathrm{e}^{\mathrm{i} \sigma T_{2}} .
\end{aligned}
$$

We exploit the conservative nature of the problem by imposing that (65) and (66) be derivable from the Lagrangian expressed by (41)-(43) and

$$
\begin{aligned}
V^{(\sigma)}= & -\left(K_{1} A_{n}^{2} \bar{A}_{n} \bar{A}_{m} \mathrm{e}^{\mathrm{i} \sigma T_{2}}+K_{2} \bar{A}_{m}^{2} A_{m} A_{n} \mathrm{e}^{\mathrm{i} \sigma T_{2}}\right. \\
& \left.+K_{3} A_{n}^{2} \bar{A}_{m}^{2} \mathrm{e}^{2 \mathrm{i} \sigma T_{2}}\right)+\mathrm{cc} .
\end{aligned}
$$

In addition to the resonance-independent symmetry condition (46) $\left(K_{m n}=K_{n m}\right)$, we obtain the resonance-dependent conditions

$$
\begin{aligned}
& 2 K_{1}=R_{2}=2 R_{4}=2 R_{5}, \quad 2 K_{2}=R_{6}=2 R_{1}=2 R_{7}, \\
& \quad \text { and } \quad 2 K_{3}=R_{3}=R_{8} .
\end{aligned}
$$

The coefficients $K_{h h}$ are given by (58), $K_{m n}$ is given by (59) and the resonance-dependent interaction coefficients are expressed as

$$
\begin{aligned}
K_{1}= & \sum_{j=1}^{\infty}\left[\left(\Lambda_{n m j}+\Lambda_{n j m}\right) \frac{\Lambda_{j n n}}{\omega_{j}^{2}-4 \omega_{n}^{2}}\right. \\
& \left.+\left(\Lambda_{n n j}+\Lambda_{n j n}\right) \frac{\Lambda_{j m n}+\Lambda_{j n m}}{\omega_{j}^{2}}\right] \\
& +\Gamma_{n n n m}+\Gamma_{n n m n}+\Gamma_{n m n n} \\
K_{2}= & \sum_{j=1}^{\infty}\left[\left(\Lambda_{n m j}+\Lambda_{n j m}\right)\left(\frac{2}{\omega_{j}^{2}}+\frac{1}{\omega_{j}^{2}-4 \omega_{m}^{2}}\right) \Lambda_{j m m}\right] \\
& +3 \Gamma_{n m m m},
\end{aligned}
$$

$$
\begin{aligned}
2 K_{3}= & \sum_{j=1}^{\infty}\left[\left(\Lambda_{n n j}+\Lambda_{n j n}\right) \frac{\Lambda_{j m m}}{\omega_{j}^{2}-4 \omega_{m}^{2}}\right. \\
& \left.+\left(\Lambda_{n m j}+\Lambda_{n j m}\right) \frac{\Lambda_{j m n}+\Lambda_{j n m}}{\omega_{j}^{2}}\right] \\
& +\Gamma_{n m m n}+\Gamma_{n m n m}+\Gamma_{n n m m} .
\end{aligned}
$$

The displacement field is given by (49) with the same second-order functions governing the displacement in the case of a three-to-one internal resonance.

\section{Direct approach}

In this section, we attack directly the equations of motion and boundary conditions with the method of multiple scales. We seek uniform expansions of $u$ and $v$ in the form

$$
\begin{aligned}
& u(x, t)=\sum_{k=1}^{3} \varepsilon^{k} u_{k}\left(x, T_{0}, T_{1}, T_{2}\right)+\cdots \\
& v(x, t)=\sum_{k=1}^{3} \varepsilon^{k} v_{k}\left(x, T_{0}, T_{1}, T_{2}\right)+\cdots
\end{aligned}
$$

with $\varepsilon$ possessing the same meaning as in the preceding sections.

Substituting (72) into the scalar form of the equations of motion (4) and boundary conditions (2), using the independence of the time scales, and equating coefficients of like powers of $\varepsilon$ yields

Order $\varepsilon$ :

$$
\begin{aligned}
& D_{0} u_{1}-v_{1}=0 \\
& D_{0} v_{1}+L u_{1}=0 \\
& \quad \text { Order } \varepsilon^{2}: \\
& D_{0} u_{2}-v_{2}=-D_{1} u_{1}, \\
& D_{0} v_{2}+L u_{2}=-D_{1} v_{1}+G_{2}\left(u_{1}, u_{1}\right) ; \\
& \quad \text { Order } \varepsilon^{3}:
\end{aligned}
$$

$D_{0} u_{3}-v_{3}=-D_{2} u_{1}-D_{1} u_{2}$,

$$
\begin{aligned}
D_{0} v_{3}+L u_{3}= & -D_{2} v_{1}-D_{1} v_{2}+G_{2}\left(u_{1}, u_{2}\right) \\
& +G_{2}\left(u_{2}, u_{1}\right)+G_{3}\left(u_{1}, u_{1}, u_{1}\right) .
\end{aligned}
$$

The boundary conditions are given by $(2)$ at all orders. 
Because the $m$ th and $n$th modes are the only modes involved in the internal resonances, we express the solution of (73) and (74) as

$$
\begin{aligned}
u_{1}= & A_{m}\left(T_{1}, T_{2}\right) \mathrm{e}^{\mathrm{i} \omega_{m} T_{0}} \phi_{m}(x) \\
& +A_{n}\left(T_{1}, T_{2}\right) \mathrm{e}^{\mathrm{i} \omega_{n} T_{0}} \phi_{n}(x)+\text { cc }
\end{aligned}
$$

and

$$
\begin{aligned}
v_{1}= & \mathrm{i} \omega_{m} A_{m}\left(T_{1}, T_{2}\right) \mathrm{e}^{\mathrm{i} \omega_{m} T_{0}} \phi_{m}(x) \\
& +\mathrm{i} \omega_{n} A_{n}\left(T_{1}, T_{2}\right) \mathrm{e}^{\mathrm{i} \omega_{n} T_{0}} \phi_{n}(x)+c c .
\end{aligned}
$$

Substituting (79) and (80) into (75) and (76) yields

$$
\begin{aligned}
D_{0} u_{2}-v_{2}= & -\left(D_{1} A_{m}\right) \mathrm{e}^{\mathrm{i} \omega_{m} T_{0}} \phi_{m} \\
& -\left(D_{1} A_{n}\right) \mathrm{e}^{\mathrm{i} \omega_{n} T_{0}} \phi_{n}+\text { cc, }
\end{aligned}
$$

$$
\begin{aligned}
D_{0} v_{2} & +L u_{2} \\
= & -\mathrm{i} \omega_{m}\left(D_{1} A_{m}\right) \mathrm{e}^{\mathrm{i} \omega_{m} T_{0}} \phi_{m} \\
& -\mathrm{i} \omega_{n}\left(D_{1} A_{n}\right) \mathrm{e}^{\mathrm{i} \omega_{n} T_{0}} \phi_{n} \\
& +\left[A_{m}^{2} \mathrm{e}^{2 \mathrm{i} \omega_{m} T_{0}}+A_{m} \bar{A}_{m}\right] G_{2}\left(\phi_{m}, \phi_{m}\right) \\
& +\left[A_{m} A_{n} \mathrm{e}^{\mathrm{i}\left(\omega_{m}+\omega_{n}\right) T_{0}}+A_{n} \bar{A}_{m} \mathrm{e}^{\mathrm{i}\left(\omega_{n}-\omega_{m}\right) T_{0}}\right] \\
& \times\left[G_{2}\left(\phi_{m}, \phi_{n}\right)+G_{2}\left(\phi_{n}, \phi_{m}\right)\right] \\
& +\left[A_{n}^{2} \mathrm{e}^{2 \mathrm{i} \omega_{n} T_{0}}+A_{n} \bar{A}_{n}\right] G_{2}\left(\phi_{n}, \phi_{n}\right)+c c .
\end{aligned}
$$

The analysis here performed is common to the considered internal resonances. In the next sections, we treat separately the cases of two-to-one, three-to-one, and one-to-one internal resonances.

\subsection{Two-to-one internal resonances}

Due to the two-to-one ratio between the frequencies of the $m$ th and $n$th modes, resonant terms may arise at second order; namely,

$$
\begin{aligned}
& D_{0} u_{2}-v_{2} \\
& =-\left(D_{1} A_{m}\right) \mathrm{e}^{\mathrm{i} \omega_{m} T_{0}} \phi_{m}-\left(D_{1} A_{n}\right) \mathrm{e}^{\mathrm{i} \omega_{n} T_{0}} \phi_{n}+c c,
\end{aligned}
$$

$$
\begin{aligned}
D_{0} v_{2} & +L u_{2} \\
= & -\mathrm{i} \omega_{m}\left(D_{1} A_{m}\right) \mathrm{e}^{\mathrm{i} \omega_{m} T_{0}} \phi_{m}-\mathrm{i} \omega_{n}\left(D_{1} A_{n}\right) \mathrm{e}^{\mathrm{i} \omega_{n} T_{0}} \phi_{n} \\
& +A_{m}^{2} \mathrm{e}^{\mathrm{i} \omega_{n} T_{0}} \mathrm{e}^{-\mathrm{i} \sigma T_{1}} G_{2}\left(\phi_{m}, \phi_{m}\right)+A_{n} \bar{A}_{m} \mathrm{e}^{\mathrm{i} \omega_{m} T_{0}} \mathrm{e}^{\mathrm{i} \sigma T_{1}}
\end{aligned}
$$

$$
\times\left[G_{2}\left(\phi_{m}, \phi_{n}\right)+G_{2}\left(\phi_{n}, \phi_{m}\right)\right]+\mathrm{cc}+\mathrm{NST} .
$$

Because the associated homogeneous problem admits non-trivial solutions (i.e., the eigensolutions), the second-order problem is solvable only if solvability conditions are satisfied. To this end, the right-hand sides of (83) and (84) are required to be orthogonal to every solution of the homogeneous adjoint problem. That is, we impose the orthogonality of the resonant inhomogeneous terms to $\left[\mathrm{i} \omega_{m}, 1\right] \exp \left(-\mathrm{i} \omega_{m} T_{0}\right) \phi_{m}$ and to $\left[\mathrm{i} \omega_{n}, 1\right] \exp \left(-\mathrm{i} \omega_{n} T_{0}\right) \phi_{n}$.

Imposing these conditions and accounting for the definition of $\Lambda_{k i j}$ provided in (6), we obtain the same solvability conditions as those obtained with the discretization approach; namely (23) and (24). Thereafter, solving these equations for $D_{1} A_{m}$ and $D_{1} A_{n}$ and substituting the results into the second-order equations (81) and (82), we obtain

$$
\begin{aligned}
D_{0} u_{2}- & v_{2}=\mathrm{i} \frac{\left(\Lambda_{m m n}+\Lambda_{m n m}\right)}{2 \omega_{m}} A_{n} \bar{A}_{m} \mathrm{e}^{\mathrm{i}\left(\omega_{n}-\omega_{m}\right) T_{0}} \phi_{m} \\
& +\mathrm{i} \frac{\Lambda_{n m m}}{2 \omega_{n}} A_{m}^{2} \mathrm{e}^{2 \mathrm{i} \omega_{m} T_{0}} \phi_{n}+\mathrm{cc}, \\
D_{0} v_{2}+L u_{2} & \\
= & A_{m}^{2} \mathrm{e}^{2 \mathrm{i} \omega_{m} T_{0}}\left[G_{2}\left(\phi_{m}, \phi_{m}\right)-\frac{1}{2} \Lambda_{n m m} \phi_{n}\right] \\
& +A_{n}^{2} \mathrm{e}^{2 \mathrm{i} \omega_{n} T_{0}} G_{2}\left(\phi_{n}, \phi_{n}\right) \\
& +A_{m} A_{n} \mathrm{e}^{\mathrm{i}\left(\omega_{m}+\omega_{n}\right) T_{0}}\left[G_{2}\left(\phi_{m}, \phi_{n}\right)\right. \\
& \left.+G_{2}\left(\phi_{n}, \phi_{m}\right)\right]+A_{n} \bar{A}_{m} \mathrm{e}^{\mathrm{i}\left(\omega_{n}-\omega_{m}\right) T_{0}} \\
& \times\left[G_{2}\left(\phi_{m}, \phi_{n}\right)+G_{2}\left(\phi_{n}, \phi_{m}\right)\right. \\
& \left.-\frac{1}{2}\left(\Lambda_{m m n}+\Lambda_{m n m}\right) \phi_{m}\right]+A_{m} \bar{A}_{m} G_{2}\left(\phi_{m}, \phi_{m}\right) \\
& +A_{n} \bar{A}_{n} G_{2}\left(\phi_{n}, \phi_{n}\right)+\mathrm{cc} .
\end{aligned}
$$

Based on the right-hand sides of (85) and (86), the second-order solution can be assumed in the form

$$
\begin{aligned}
u_{2}= & A_{m}^{2} \mathrm{e}^{2 \mathrm{i} \omega_{m} T_{0}} \Psi_{m m}(x)+A_{m} \bar{A}_{m} \chi_{m m}(x) \\
& +A_{n}^{2} \mathrm{e}^{2 \mathrm{i} \omega_{n} T_{0}} \Psi_{n n}(x)+A_{n} \bar{A}_{n} \chi_{n n}(x) \\
& +A_{n} A_{m} \mathrm{e}^{\mathrm{i}\left(\omega_{n}+\omega_{m}\right) T_{0}} \Psi_{m n}(x) \\
& +A_{n} \bar{A}_{m} \mathrm{e}^{\mathrm{i}\left(\omega_{n}-\omega_{m}\right) T_{0}} \chi_{m n}(x)+c c,
\end{aligned}
$$




$$
\begin{aligned}
v_{2}= & A_{m}^{2} \mathrm{e}^{2 \mathrm{i} \omega_{m} T_{0}} \eta_{m m}(x)+A_{m} \bar{A}_{m} \zeta_{m m}(x) \\
& +A_{n}^{2} \mathrm{e}^{2 \mathrm{i} \omega_{n} T_{0}} \eta_{n n}(x)+A_{n} \bar{A}_{n} \zeta_{n n}(x) \\
& +A_{n} A_{m} \mathrm{e}^{\mathrm{i}\left(\omega_{n}+\omega_{m}\right) T_{0}} \eta_{m n}(x) \\
& +A_{n} \bar{A}_{m} \mathrm{e}^{\mathrm{i}\left(\omega_{n}-\omega_{m}\right) T_{0}} \zeta_{m n}(x)+c c .
\end{aligned}
$$

Substituting (87) and (88) into (85) and (86), we obtain a set of coupled boundary-value problems in the unknown functions $\Psi_{k k}\left(\chi_{k k}\right)$ and $\eta_{k k}\left(\zeta_{k k}\right)$ which, in turn, can be decoupled yielding

$$
\begin{aligned}
& L \Psi_{m m}-\omega_{n}^{2} \Psi_{m m}= G_{2}\left(\phi_{m}, \phi_{m}\right)-\Lambda_{n m m} \phi_{n} \\
& L \chi_{m n}-\omega_{m}^{2} \chi_{m n}= G_{2}\left(\phi_{m}, \phi_{n}\right)+G_{2}\left(\phi_{n}, \phi_{m}\right) \\
&-\left(\Lambda_{m m n}+\Lambda_{m n m}\right) \phi_{m}, \\
& L \Psi_{n n}-4 \omega_{n}^{2} \Psi_{n n}= G_{2}\left(\phi_{n}, \phi_{n}\right), \\
& L \Psi_{m n}-\left(\omega_{m}+\omega_{n}\right)^{2} \Psi_{m n} \\
&=G_{2}\left(\phi_{m}, \phi_{n}\right)+G_{2}\left(\phi_{n}, \phi_{m}\right)
\end{aligned}
$$

$$
L \chi_{m m}=G_{2}\left(\phi_{m}, \phi_{m}\right), \quad L \chi_{n n}=G_{2}\left(\phi_{n}, \phi_{n}\right),
$$

with all of the functions satisfying the boundary conditions (2). On the other hand, the functions associated with the second-order velocity field are given by

$$
\begin{aligned}
& \eta_{m m}=2 \mathrm{i} \omega_{m} \Psi_{m m}-\mathrm{i} \frac{\Lambda_{n m m}}{2 \omega_{n}} \phi_{n}, \\
& \zeta_{m n}=\mathrm{i}\left(\omega_{n}-\omega_{m}\right) \chi_{m n}-\mathrm{i} \frac{\Lambda_{m m n}+\Lambda_{m n m}}{2 \omega_{m}} \phi_{m}, \\
& \eta_{n n}=2 \mathrm{i} \omega_{n} \Psi_{n n}, \quad \eta_{m n}=\mathrm{i}\left(\omega_{m}+\omega_{n}\right) \Psi_{m n}, \\
& \zeta_{n n}=\zeta_{m m}=0 .
\end{aligned}
$$

The solutions of the boundary-value problems (91) -(93) are uniquely determined; however, because the homogeneous problems associated with (89) and (90) admit non-trivial solutions ( $\phi_{n}$ and $\phi_{m}$, respectively), their solutions are uniquely determined by imposing the orthogonality of $\left[\Psi_{m m}, \eta_{m m}\right]$ to the adjoint solution $\left[\mathrm{i} \omega_{n}, 1\right] \phi_{n}$ and that of $\left[\chi_{m n}, \zeta_{m n}\right]$ to the adjoint solution $\left[\mathrm{i} \omega_{m}, 1\right] \phi_{m}$, respectively. Using the modal expansion method, we can express the sought solutions as

$$
\begin{aligned}
\Psi_{m m}= & \sum_{k=1, k \neq n}^{\infty} \frac{\Lambda_{k m m}}{\omega_{k}^{2}-\omega_{n}^{2}} \phi_{k}(x)+\frac{\Lambda_{n m m}}{4 \omega_{n}^{2}} \phi_{n}(x), \\
\eta_{m m}= & \mathrm{i} \omega_{n} \sum_{k=1, k \neq n}^{\infty} \frac{\Lambda_{k m m}}{\omega_{k}^{2}-\omega_{n}^{2}} \phi_{k}(x) \\
& -\mathrm{i} \omega_{n}\left[\frac{\Lambda_{n m m}}{4 \omega_{n}^{2}} \phi_{n}(x)\right], \\
\chi_{m n}= & \sum_{k=1, k \neq m}^{\infty} \frac{\Lambda_{k m n}+\Lambda_{k n m}}{\omega_{k}^{2}-\omega_{m}^{2}} \phi_{k}(x) \\
& +\frac{\Lambda_{m m n}+\Lambda_{m n m}}{4 \omega_{m}^{2}} \phi_{m}(x), \\
\eta_{m n}= & \mathrm{i} \omega_{m} \sum_{k=1, k \neq m}^{\infty} \frac{\Lambda_{k m n}+\Lambda_{k n m}}{\omega_{k}^{2}-\omega_{m}^{2}} \phi_{k}(x) \\
& -\mathrm{i} \omega_{m}\left[\frac{\Lambda_{m m n}+\Lambda_{m n m}}{4 \omega_{m}^{2}} \phi_{m}(x)\right] .
\end{aligned}
$$

Comparing (50) with (97) and (51) with (99), we conclude that the functions $\Psi_{m m}$ and $\chi_{m n}$ obtained with the direct approach are the same as $\psi_{m m}$ and $\kappa_{m n}$ obtained with the full-basis discretization approach. It can also be proved, using a technique similar to that used in [14], that the remaining functions $\Psi_{n n}, \Psi_{m n}, \chi_{n n}$, and $\chi_{m m}$, obtained with the direct approach, are, respectively, the same as $\psi_{n n}, \psi_{m n}, \kappa_{n n}$, and $\kappa_{m m}$, obtained with the discretization approach, respectively.

Substituting (79), (80), (87), and (88) into the third-order problem yields

$$
\begin{aligned}
D_{0} u_{3} & -v_{3} \\
= & -\left(D_{2} A_{m}\right) \mathrm{e}^{\mathrm{i} \omega_{m} T_{0}} \phi_{m}-\left(D_{2} A_{n}\right) \mathrm{e}^{\mathrm{i} \omega_{n} T_{0}} \phi_{n} \\
& -D_{1}\left(A_{m}^{2} \mathrm{e}^{-\mathrm{i} \sigma T_{1}}\right) \mathrm{e}^{\mathrm{i} \omega_{n} T_{0}} \Psi_{m m} \\
& -D_{1}\left(A_{n} \bar{A}_{m} \mathrm{e}^{\mathrm{i} \sigma T_{1}}\right) \mathrm{e}^{\mathrm{i} \omega_{m} T_{0}} \chi_{m n}+\mathrm{cc}+\mathrm{NST}, \quad(101) \\
D_{0} v_{3} & +L u_{3} \\
= & -\mathrm{i} \omega_{m}\left(D_{2} A_{m}\right) \mathrm{e}^{\mathrm{i} \omega_{m} T_{0}} \phi_{m}-\mathrm{i} \omega_{n}\left(D_{2} A_{n}\right) \mathrm{e}^{\mathrm{i} \omega_{n} T_{0}} \phi_{n} \\
& -D_{1}\left(A_{m}^{2} \mathrm{e}^{-\mathrm{i} \sigma T_{1}}\right) \mathrm{e}^{\mathrm{i} \omega_{n} T_{0}} \eta_{m m} \\
& -D_{1}\left(A_{n} \bar{A}_{m} \mathrm{e}^{\mathrm{i} \sigma T_{1}}\right) \mathrm{e}^{\mathrm{i} \omega_{m} T_{0}} \zeta_{m n}
\end{aligned}
$$




$$
\begin{aligned}
& +A_{m}^{2} \bar{A}_{m} \mathrm{e}^{\mathrm{i} \omega_{m} T_{0}}\left[G_{2}\left(\Psi_{m m}, \phi_{m}\right)+G_{2}\left(\phi_{m}, \Psi_{m m}\right)\right. \\
& +2 G_{2}\left(\phi_{m}, \chi_{m m}\right)+2 G_{2}\left(\chi_{m m}, \phi_{m}\right) \\
& \left.+3 G_{3}\left(\phi_{m}, \phi_{m}, \phi_{m}\right)\right] \\
& +A_{m} A_{n} \bar{A}_{n} \mathrm{e}^{\mathrm{i} \omega_{m} T_{0}}\left[G_{2}\left(\Psi_{m n}, \phi_{n}\right)\right. \\
& +G_{2}\left(\phi_{n}, \Psi_{m n}\right)+G_{2}\left(\chi_{m n}, \phi_{n}\right) \\
& +G_{2}\left(\phi_{n}, \chi_{m n}\right)+2 G_{2}\left(\chi_{n n}, \phi_{m}\right)+2 G_{2}\left(\phi_{m}, \chi_{n n}\right) \\
& +2 G_{3}\left(\phi_{m}, \phi_{n}, \phi_{n}\right)+2 G_{3}\left(\phi_{n}, \phi_{m}, \phi_{n}\right) \\
& \left.+2 G_{3}\left(\phi_{n}, \phi_{n}, \phi_{m}\right)\right] \\
& +A_{n}^{2} \bar{A}_{n} \mathrm{e}^{\mathrm{i} \omega_{n} T_{0}}\left[G_{2}\left(\Psi_{n n}, \phi_{n}\right)+G_{2}\left(\phi_{n}, \Psi_{n n}\right)\right. \\
& +2 G_{2}\left(\phi_{n}, \chi_{n n}\right)+2 G_{2}\left(\chi_{n n}, \phi_{n}\right) \\
& \left.+3 G_{3}\left(\phi_{n}, \phi_{n}, \phi_{n}\right)\right] \\
& +A_{n} A_{m} \bar{A}_{m} \mathrm{e}^{\mathrm{i} \omega_{n} T_{0}}\left[G_{2}\left(\Psi_{m n}, \phi_{m}\right)+G_{2}\left(\phi_{m}, \Psi_{m n}\right)\right. \\
& +G_{2}\left(\chi_{m n}, \phi_{m}\right)+G_{2}\left(\phi_{m}, \chi_{m n}\right)+2 G_{2}\left(\chi_{m m}, \phi_{n}\right) \\
& +2 G_{2}\left(\phi_{n}, \chi_{m m}\right)+2 G_{3}\left(\phi_{m}, \phi_{m}, \phi_{n}\right) \\
& \left.+2 G_{3}\left(\phi_{m}, \phi_{n}, \phi_{m}\right)+2 G_{3}\left(\phi_{n}, \phi_{m}, \phi_{m}\right)\right] \\
& +\mathrm{cc}+\mathrm{NST} .
\end{aligned}
$$

Because the associated homogeneous problem admits non-trivial solutions, the third-order problem is solvable only if the right-hand sides of (101) and (102) are orthogonal to every solution of the adjoint homogeneous problem. The solvability conditions are formally the same as (34) and (35), obtained with the discretization approach, except for the fact that the coefficients are now expressed as

$$
\begin{aligned}
K_{h h}= & \left\langle\phi_{h} G_{2}\left(\phi_{h}, \Psi_{h h}\right)\right\rangle+\left\langle\phi_{h} G_{2}\left(\Psi_{h h}, \phi_{h}\right)\right\rangle \\
& +2\left\langle\phi_{h} G_{2}\left(\phi_{h}, \chi_{h h}\right)\right\rangle+2\left\langle\phi_{h} G_{2}\left(\chi_{h h}, \phi_{h}\right)\right\rangle \\
& +3\left\langle\phi_{h} G_{3}\left(\phi_{h}, \phi_{h}, \phi_{h}\right)\right\rangle, \quad h=m, n, \quad(103) \\
K_{m n}= & \left\langle\phi_{m} G_{2}\left(\phi_{n}, \Psi_{m n}\right)\right\rangle+\left\langle\phi_{m} G_{2}\left(\Psi_{m n}, \phi_{n}\right)\right\rangle \\
& +\left\langle\phi_{m} G_{2}\left(\phi_{n}, \chi_{m n}\right)\right\rangle+\left\langle\phi_{m} G_{2}\left(\chi_{m n}, \phi_{n}\right)\right\rangle \\
& +2\left\langle\phi_{m} G_{2}\left(\phi_{m}, \chi_{n n}\right)\right\rangle+2\left\langle\phi_{m} G_{2}\left(\chi_{n n}, \phi_{m}\right)\right\rangle \\
& +2\left\langle\phi_{m} G_{3}\left(\phi_{n}, \phi_{n}, \phi_{m}\right)\right\rangle+2\left\langle\phi_{m} G_{3}\left(\phi_{n}, \phi_{m}, \phi_{n}\right)\right\rangle \\
& +2\left\langle\phi_{m} G_{3}\left(\phi_{m}, \phi_{n}, \phi_{n}\right)\right\rangle .
\end{aligned}
$$

These coefficients can be shown to be the same as those obtained with the discretization approach [14].

The displacement field can be expressed as in (49) with all the $\Psi$ 's and the $\chi$ 's substituting the $\psi$ 's and the $\kappa$ 's, respectively. Therefore, having established the equivalence of the functions $\Psi$ 's and $\chi$ 's with $\psi$ 's and $\kappa$ 's, respectively; having established the equivalence of the modulation equations obtained with both approaches, we conclude that the approximation of the resonant non-linear normal modes obtained with the direct approach is the same as that obtained with the full-basis discretization approach. This result is general and holds also for a three-to-one and a one-to-one internal resonance.

\subsection{Three-to-one internal resonances}

Because resonant terms do not arise at second order, the solvability conditions for the second-order problem, (75) and (76), require that $D_{1} A_{m}=0$ and $D_{1} A_{n}=0$. Therefore, as expected, the amplitudes and phases of the interacting modes do not depend on the scale $T_{1}$. Then, the solutions of (75) and (76) can be expressed as

$$
\begin{aligned}
u_{2}= & A_{m}^{2} \mathrm{e}^{2 \mathrm{i} \omega_{m} T_{0}} \Psi_{m m}(x)+A_{m} \bar{A}_{m} \chi_{m m}(x) \\
& +A_{n}^{2} \mathrm{e}^{2 \mathrm{i} \omega_{n} T_{0}} \Psi_{n n}(x)+A_{n} \bar{A}_{n} \chi_{n n}(x) \\
& +A_{n} A_{m} \mathrm{e}^{\mathrm{i}\left(\omega_{n}+\omega_{m}\right) T_{0}} \Psi_{m n}(x) \\
& +A_{n} \bar{A}_{m} \mathrm{e}^{\mathrm{i}\left(\omega_{n}-\omega_{m}\right) T_{0}} \chi_{m n}(x)+\mathrm{cc}, \\
v_{2}= & A_{m}^{2} \mathrm{e}^{2 \mathrm{i} \omega_{m} T_{0}} \eta_{m m}(x)+A_{m} \bar{A}_{m} \zeta_{m m}(x) \\
& +A_{n}^{2} \mathrm{e}^{2 \mathrm{i} \omega_{n} T_{0}} \eta_{n n}(x)+A_{n} \bar{A}_{n} \zeta_{n n}(x) \\
& +A_{n} A_{m} \mathrm{e}^{\mathrm{i}\left(\omega_{n}+\omega_{m}\right) T_{0}} \eta_{m n}(x) \\
& +A_{n} \bar{A}_{m} \mathrm{e}^{\mathrm{i}\left(\omega_{n}-\omega_{m}\right) T_{0}} \zeta_{m n}(x)+\mathrm{cc} .
\end{aligned}
$$

The second-order shape functions $\Psi_{n n}, \Psi_{m n}, \chi_{m m}$, and $\chi_{n n}$ are solutions of the boundary-value problems (91)-(93). On the other hand, $\Psi_{m m}$ and $\chi_{m n}$ are now obtained as solutions of the following modified boundary-value problems:

$$
\begin{aligned}
& L \Psi_{m m}-4 \omega_{m}^{2} \Psi_{m m}=G_{2}\left(\phi_{m}, \phi_{m}\right), \\
& L \chi_{m n}-\left(\omega_{n}-\omega_{m}\right)^{2} \chi_{m n} \\
& =G_{2}\left(\phi_{m}, \phi_{n}\right)+G_{2}\left(\phi_{n}, \phi_{m}\right),
\end{aligned}
$$


with $\Psi_{m m}$ and $\chi_{m n}$ satisfying the boundary conditions (2).

Substituting (79), (80), (105), and (106) into (77) and (78), resonant terms arise depending on the type of internal resonance. Imposing solvability conditions, we obtain the modulation equations with the same structure as (56) and (57). The pertinent resonance-independent coefficients have the expressions given by (103) and (104) and must be evaluated using the newly defined functions. On the other hand, the interaction coefficients $\Lambda_{m}$ and $\Lambda_{n}$ are given by

$$
\begin{aligned}
\Lambda_{m}= & \left\langle\phi_{m} G_{2}\left(\phi_{n}, \Psi_{m m}\right)\right\rangle+\left\langle\phi_{m} G_{2}\left(\Psi_{m m}, \phi_{n}\right)\right\rangle \\
& +\left\langle\phi_{m} G_{2}\left(\phi_{m}, \chi_{m n}\right)\right\rangle+\left\langle\phi_{m} G_{2}\left(\chi_{m n}, \phi_{m}\right)\right\rangle \\
& +\left\langle\phi_{m} G_{3}\left(\phi_{n}, \phi_{m}, \phi_{m}\right)\right\rangle+\left\langle\phi_{m} G_{3}\left(\phi_{m}, \phi_{n}, \phi_{m}\right)\right\rangle \\
& +\left\langle\phi_{m} G_{3}\left(\phi_{m}, \phi_{m}, \phi_{n}\right)\right\rangle,
\end{aligned}
$$$$
\Lambda_{n}=\left\langle\phi_{n} G_{2}\left(\phi_{m}, \Psi_{m m}\right)\right\rangle+\left\langle\phi_{n} G_{2}\left(\Psi_{m m}, \phi_{m}\right)\right\rangle
$$$$
+\left\langle\phi_{n} G_{3}\left(\phi_{m}, \phi_{m}, \phi_{m}\right)\right\rangle \text {. }
$$

\subsection{One-to-one internal resonances}

The analysis performed for the three-to-one internal resonance holds for the one-to-one internal resonance up to second order. The solvability conditions at third-order yield the modulation equations with the same structure as (65) and (66). The resonance-independent coefficients have the expressions given by (103) and (104) whereas the resonance-dependent interaction coefficients are now expressed as

$$
\begin{aligned}
K_{1}= & \left\langle\phi_{n} G_{2}\left(\phi_{m}, \Psi_{n n}\right)\right\rangle+\left\langle\phi_{n} G_{2}\left(\Psi_{n n}, \phi_{m}\right)\right\rangle \\
& +\left\langle\phi_{n} G_{2}\left(\phi_{n}, \chi_{m n}\right)\right\rangle+\left\langle\phi_{n} G_{2}\left(\chi_{m n}, \phi_{n}\right)\right\rangle \\
& +\left\langle\phi_{n} G_{3}\left(\phi_{n}, \phi_{n}, \phi_{m}\right)\right\rangle+\left\langle\phi_{n} G_{3}\left(\phi_{n}, \phi_{m}, \phi_{n}\right)\right\rangle \\
& +\left\langle\phi_{n} G_{3}\left(\phi_{m}, \phi_{n}, \phi_{n}\right)\right\rangle
\end{aligned}
$$

$$
\begin{aligned}
K_{2}= & \left\langle\phi_{n} G_{2}\left(\phi_{m}, \Psi_{m m}\right)\right\rangle+\left\langle\phi_{n} G_{2}\left(\Psi_{m m}, \phi_{m}\right)\right\rangle \\
& +2\left\langle\phi_{n} G_{2}\left(\phi_{m}, \chi_{m m}\right)\right\rangle+2\left\langle\phi_{n} G_{2}\left(\chi_{m m}, \phi_{m}\right)\right\rangle \\
& +3\left\langle\phi_{n} G_{3}\left(\phi_{m}, \phi_{m}, \phi_{m}\right)\right\rangle,
\end{aligned}
$$

$$
\begin{aligned}
2 K_{3}= & \left\langle\phi_{n} G_{2}\left(\phi_{n}, \Psi_{m m}\right)\right\rangle+\left\langle\phi_{n} G_{2}\left(\Psi_{m m}, \phi_{n}\right)\right\rangle \\
& +\left\langle\phi_{n} G_{2}\left(\phi_{m}, \chi_{m n}\right)\right\rangle+\left\langle\phi_{n} G_{2}\left(\chi_{m n}, \phi_{m}\right)\right\rangle \\
& +\left\langle\phi_{n} G_{3}\left(\phi_{m}, \phi_{m}, \phi_{n}\right)\right\rangle+\left\langle\phi_{n} G_{3}\left(\phi_{m}, \phi_{n}, \phi_{m}\right)\right\rangle \\
& +\left\langle\phi_{n} G_{3}\left(\phi_{n}, \phi_{m}, \phi_{m}\right)\right\rangle .
\end{aligned}
$$

\section{Coupled/uncoupled non-linear normal modes. Non-linear orthogonality}

In the next sections, we discuss conditions for the existence of coupled/uncoupled non-linear normal modes and the associated stability for each internal resonance condition. Moreover, we discuss necessary and sufficient conditions for the occurrence of non-linear orthogonality between the modes. To this end, we use the modulation equations governing the amplitudes and phases of the interacting modes. There are two forms of non-linear orthogonality: a local form and a global form. In the local case, there are regions in the frequency-detuning-amplitude space where coupled non-linear normal modes (multimodal periodic motions with a constant relative phase) either do not exist or they are unstable. Hence, there cannot occur periodic exchanges of energy between the modes. Of course, this circumstance does not necessarily imply absence of other forms of interaction between the modes such as quasiperiodic or non-periodic interactions. In the global form, the orthogonality is independent of the detuning, the amplitudes, and the relative phases. We show that the global conditions are an extension of the linear orthogonality concept applicable to the modes of self-adjoint systems when they enter their weak non-linear regime.

In addition, we express the displacement field of the coupled or uncoupled non-linear normal modes by incorporating the solutions of the modulation equations for each resonance condition.

\subsection{Two-to-one internal resonances}

We express $A_{j}$ in the polar form $A_{j}=(1 / 2) a_{j} \exp$ $\left(\mathrm{i} \beta_{j}\right)$, separate real and imaginary parts in the 
modulation equations (39) and (40), and obtain their real-valued form as

$$
\begin{aligned}
\dot{a}_{m}= & \frac{S}{4 \omega_{m}} a_{m} a_{n} \sin \gamma \\
a_{m} \dot{\beta}_{m}= & -\frac{S}{4 \omega_{m}} a_{m} a_{n} \cos \gamma-\frac{K_{m m}}{8 \omega_{m}} a_{m}^{3} \\
& \quad-\frac{K_{m n}}{8 \omega_{m}} a_{m} a_{n}^{2}, \\
\dot{a}_{n}=- & \frac{S}{8 \omega_{n}} a_{m}^{2} \sin \gamma, \\
a_{n} \dot{\beta}_{n}= & -\frac{S}{8 \omega_{n}} a_{m}^{2} \cos \gamma-\frac{K_{n n}}{8 \omega_{n}} a_{n}^{3}-\frac{K_{m n}}{8 \omega_{n}} a_{n} a_{m}^{2},
\end{aligned}
$$

where the relative phase between the interacting modes is given by $\gamma=\sigma t-2 \beta_{m}+\beta_{n}$.

There are two possible sets of solutions: (i) coupled resonant modes $\left(a_{m} \neq 0\right.$ and $\left.a_{n} \neq 0\right)$ and (ii) the high-frequency uncoupled mode $\left(a_{m}=0\right.$ and $\left.a_{n} \neq 0\right)$.

In the case of coupled resonant modes, because $a_{m} \neq 0$ and $a_{n} \neq 0$, we can solve for $\dot{\beta}_{m}$ and $\dot{\beta}_{n}$ and substitute the result into the equation governing the relative phase, thereby obtaining

$$
\begin{aligned}
\dot{\gamma}= & \sigma+\frac{1}{16 \omega_{m}}\left[S \cos \gamma\left(8-\frac{a_{m}^{2}}{a_{n}^{2}}\right) a_{n}\right. \\
& \left.+\left(4 K_{m n}-K_{n n}\right) a_{n}^{2}+\left(4 K_{m m}-K_{m n}\right) a_{m}^{2}\right] .
\end{aligned}
$$

Consequently, (113), (115), and (117) govern the slow amplitude- and phase-modulations of the coupled non-linear normal modes. The fixed points of these equations (i.e., $\dot{a}_{m}=\dot{a}_{n}=\dot{\gamma}=0$ ) correspond to periodic motions of the original system. These solutions are given by

$$
\begin{aligned}
\gamma= & n \pi, \quad\left(4 K_{m m}-K_{m n}\right) a_{m}^{2}+\left(4 K_{m n}-K_{n n}\right) a_{n}^{2} \\
& +S \cos \gamma\left(8-\frac{a_{m}^{2}}{a_{n}^{2}}\right) a_{n}+\left(16 \omega_{m}\right) \sigma=0 .
\end{aligned}
$$

We note that, when the approximation of the two-to-one internally resonant non-linear normal modes is truncated to second order, (113) and (115) are the same as in the first-order approximation and yield the same relative phase $\gamma=n \pi$. Consequently, the relative phase is not affected by the order of the approximation. On the contrary, (117) is affected by higher-order terms. At the same time, we note that these higher-order terms are not associated with the effects of the two-to-one internal resonance. Therefore, higher-order terms do not influence the activation of the resonance but only the detuning-amplitude relationship and the stability bounds of the ensuing solutions. Hence, to analyze conditions for the resonance activation or the dual problem of orthogonality, we consider the phase equation neglecting higher-order terms; that is,

$\dot{\gamma}=\sigma+\frac{S \cos \gamma}{16 \omega_{m}}\left(8-\frac{a_{m}^{2}}{a_{n}^{2}}\right) a_{n}$

and the associated detuning-amplitude equation

$$
\begin{aligned}
& S \cos \gamma\left(\frac{a_{m}}{a_{n}}\right)^{2} \\
& \quad-8\left[S \cos \gamma+2 \omega_{m}\left(\frac{\sigma}{a_{n}}\right)\right]=0 .
\end{aligned}
$$

Eq. (120), when $S \neq 0$, possesses the following real solutions:

$$
\begin{aligned}
& \text { (a): } c= \pm 2 \sqrt{2}\left[1+\frac{\hat{\sigma}}{\hat{\sigma}_{s}}\right]^{1 / 2}, \quad \gamma=2 n \pi, \\
& \text { (b): } c= \pm 2 \sqrt{2}\left[1-\frac{\hat{\sigma}}{\hat{\sigma}_{s}}\right]^{1 / 2}, \quad \gamma=(2 n+1) \pi,
\end{aligned}
$$

where $c=a_{m} / a_{n}, \hat{\sigma}=\sigma / a_{n}, \hat{\sigma}_{s}=S /\left(2 \omega_{m}\right)$, and $n$ is an arbitrary integer number. The coupled mode (a) exists for $\hat{\sigma} \gtrless \mp\left|\hat{\sigma}_{s}\right|$ if $S \gtrless 0$. On the other hand, the coupled mode (b) exists for $\hat{\sigma} \lessgtr \pm\left|\hat{\sigma}_{s}\right|$ if $S \gtrless 0$.

We compute the eigenvalues of the Jacobian matrix of the right-hand sides of (113), (115), and (119). One of the eigenvalues is zero (the system is undamped) and the other two eigenvalues are

$\lambda= \pm \mathrm{i} \frac{\left|\hat{\sigma}_{s}\right|}{8} a_{m}\left[c^{2}+16\right]^{1 / 2}$.

Therefore, the coupled non-linear normal modes are marginally stable (centers) [24] for any value of the internal detuning.

The displacement field, to second order, on account of the solutions for $\beta_{m}$ and $\beta_{n}$, can be 
expressed as

$$
\begin{aligned}
u(x, t) & \\
= & a_{m} \cos \left(\omega_{m N} t+\beta_{m 0}\right) \phi_{m}(x) \\
& +a_{n} \cos \left[2\left(\omega_{m N} t+\beta_{m 0}\right)+\gamma\right] \phi_{n}(x) \\
& +\frac{1}{2}\left\{a_{m}^{2}\left[\cos \left[2\left(\omega_{m N} t+\beta_{m 0}\right)\right] \Psi_{m m}(x)+\chi_{m m}(x)\right]\right. \\
& +a_{n}^{2}\left[\cos \left[4\left(\omega_{m N} t+\beta_{m 0}\right)+2 \gamma\right] \Psi_{n n}(x)+\chi_{n n}(x)\right] \\
& +a_{n} a_{m}\left[\cos \left[3\left(\omega_{m N} t+\beta_{m 0}\right)+\gamma\right] \Psi_{m n}(x)\right. \\
& \left.\left.+\cos \left(\omega_{m N} t+\beta_{m 0}\right) \chi_{m n}(x)\right]\right\},
\end{aligned}
$$

where

$$
\begin{aligned}
\omega_{m N}= & \omega_{m}-\left[\frac{S}{4 \omega_{m}} \cos \gamma a_{n}\right] \\
& -\frac{K_{m m}}{8 \omega_{m}} a_{m}^{2}-\frac{K_{m n}}{8 \omega_{m}} a_{n}^{2}
\end{aligned}
$$

is the non-linear frequency expansion of the $m$ th mode, $a_{m} / a_{n}$ is given (when considering the first-order solution) by (121) when $\gamma=2 n \pi$ or by (122) when $\gamma=(2 n+1) \pi$ and $\beta_{m 0}$ is a constant. Clearly, if the expansion is truncated to first order, we capture neither the second-order frequency corrections nor the spacetime corrections to the first-order displacement field. Furthermore, inspecting the displacement field (124) and the non-linear frequency (125) truncated to first order, and the structure of the solutions, (121) and (122), we conclude that the branches of the two solutions described by (121) and (122) which are antisymmetric with respect to the origin in the $c-\hat{\sigma}$ plane (i.e., putting $c \rightarrow-c$ and $\gamma \rightarrow \gamma+\pi$ ) correspond to the same coupled mode.

In the case of the uncoupled high-frequency mode ( $a_{m}=0$ and $a_{n} \neq 0$ ), putting $a_{m}=0$ in (116), we solve for $\beta_{n}$ and obtain $\beta_{n}=-K_{n n} /\left(8 \omega_{n}\right) a_{n}^{2} t+\beta_{n 0}$. We compute the eigenvalues of the uncoupled mode using the low-order expansion. To this end, we express the complex-valued amplitudes $A_{m}$ and $A_{n}$ in different coordinates [10]; that is, $A_{m}=(1 / 2)\left(p_{m}-\mathrm{i} q_{m}\right) \exp$ (is) and $A_{n}=(1 / 2) a_{n} \exp \left(\mathrm{i} \beta_{n}\right)$. We substitute these transformations into (39), determine $s$ such that the resulting equations are autonomous, linearize them, and substitute for $\dot{\beta}_{n}$. The eigenvalues of the resulting equations are

$\lambda= \pm \mathrm{i} \frac{a_{n}}{2}\left[\hat{\sigma}^{2}-\hat{\sigma}_{s}^{2}\right]^{1 / 2}$

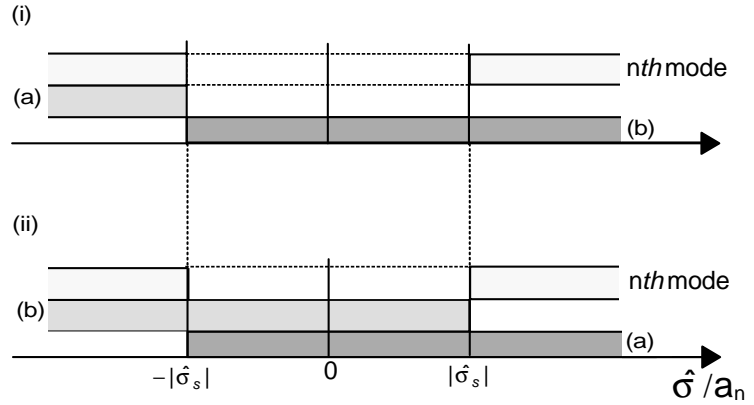

Fig. 1. Regions of existence and stability of the non-linear normal modes of type (a) and (b) due to a 2:1 internal resonance when (i) $S<0$ and (ii) $S>0$. Dashed lines indicate the unstable region of the uncoupled mode.

Therefore, the uncoupled mode is marginally stable (center) when $|\hat{\sigma}|>\left|\hat{\sigma}_{s}\right|$; it is unstable (saddle) when $|\hat{\sigma}|<\left|\hat{\sigma}_{s}\right|$; and it is degenerate (center-saddle) when $|\hat{\sigma}|=\left|\hat{\sigma}_{s}\right|$.

The approximate displacement field of the uncoupled high-frequency mode is readily expressed as

$$
\begin{aligned}
u(x, t)= & a_{n} \cos \left(\omega_{n N} t+\beta_{n 0}\right) \phi_{n}(x) \\
& +\frac{1}{2} a_{n}^{2}\left[\cos 2\left(\omega_{n N} t+\beta_{n 0}\right) \Psi_{n n}(x)\right. \\
& \left.+\chi_{n n}(x)\right],
\end{aligned}
$$

where

$\omega_{n N}=\omega_{n}-\frac{K_{n n}}{8 \omega_{n}} a_{n}^{2}$

is the expansion of the non-linear frequency of the $n$th mode.

In Fig. 1, we show the ranges of existence and stability of the normal modes based on the truncated approximation. Therefore, the system possesses either three modes (two coupled stable modes and one unstable uncoupled mode when $-\left|\hat{\sigma}_{s}\right|<\hat{\sigma}<\left|\hat{\sigma}_{s}\right|$ ) or two modes (one coupled stable mode and one stable uncoupled mode, outside of the previous range). Hence, local orthogonality between the modes never occurs for a two-to-one internal resonance.

Thus far, we have discussed the case $S \neq 0$. Next, we concentrate on the occurrence of the global non-linear orthogonality condition for the modes. The non-linear interaction coefficient governing the two-to-one interaction is $S=2 \Lambda_{\mathrm{nmm}}$. Therefore, it is easy to prove the following. 
Proposition Ia. A necessary and sufficient condition for orthogonality of the non-linear normal modes due to a two-to-one internal resonance is $S=0$.

To show the sufficient condition, we note that when $S=0$, the potential energy $V^{(\sigma)}$ resulting from the postulated interaction, given by (44), is identically zero; hence, the interaction is not activated. To show the necessary condition, we note that if the modes are orthogonal/uncoupled, no potential energy can be stored into the system as a result of an inactive interaction. Therefore, setting $V^{(\sigma)}$ to zero yields $S=0$. In conclusion, if the modes are orthogonal, necessarily $S=0$.

A mechanical interpretation of this global non-linear orthogonality condition can be sought by considering the mechanical meaning of the coefficient $\Lambda_{n m m}=\left\langle\phi_{n} G_{2}\left(\phi_{m}, \phi_{m}\right)\right\rangle=S / 2$. The latter can be interpreted as the virtual work performed by the quadratic elastic forces associated with the low-frequency mode in the displacement of the high-frequency mode. On the other hand, the linear orthogonality condition $\left(\left\langle\phi_{m} L \phi_{n}\right\rangle=0\right)$ implies that the elastic forces associated with one mode do not perform virtual work in the displacement associated with the other mode. Hence,

Proposition Ib. A necessary and sufficient condition for orthogonality of the non-linear normal modes due to a two-to-one internal resonance is that the virtual work performed by the quadratic elastic forces associated with the low-frequency mode in the displacement of the high-frequency mode be zero.

\subsection{Three-to-one internal resonances}

In this section, we follow the same line of analysis as that of the two-to-one internal resonance. Expressing $A_{j}$ in polar form, and separating real and imaginary parts in (56) and (57), we obtain

$$
\begin{aligned}
\dot{a}_{m}= & \frac{\Lambda}{8 \omega_{m}} a_{n} a_{m}^{2} \sin \gamma, \\
a_{m} \dot{\beta}_{m}= & -\frac{K_{m m}}{8 \omega_{m}} a_{m}^{3}-\frac{K_{m n}}{8 \omega_{m}} a_{m} a_{n}^{2} \\
& -\frac{\Lambda}{8 \omega_{m}} a_{n} a_{m}^{2} \cos \gamma, \\
\dot{a}_{n}=- & \frac{\Lambda}{3} \frac{1}{8 \omega_{n}} a_{m}^{3} \sin \gamma,
\end{aligned}
$$

$$
a_{n} \dot{\beta}_{n}=-\frac{K_{n n}}{8 \omega_{n}} a_{n}^{3}-\frac{K_{m n}}{8 \omega_{n}} a_{n} a_{m}^{2}-\frac{\Lambda}{24 \omega_{n}} a_{m}^{3} \cos \gamma
$$

where the relative phase between the modes is given by $\gamma=\sigma t-3 \beta_{m}+\beta_{n}$.

There are two possibilities: (i) $a_{m}=0$ and $a_{n} \neq 0$ and (ii) $a_{m} \neq 0$ and $a_{n} \neq 0$. The first case corresponds to an uncoupled non-linear normal mode whose displacement is in the form of (127). To determine the stability of this mode, we substitute the polar form for $A_{n}$ and the Cartesian form for $A_{m}$ in (56), determine $s$ such that the resulting equations are autonomous, separate the outcome into real and imaginary parts, linearize the obtained equations in $p_{m}$ and $q_{m}$, and substitute $\dot{\beta}_{n}=-K_{n n} /\left(8 \omega_{n}\right) a_{n}^{2}$ in it. The eigenvalues are $\lambda_{1,2}= \pm \frac{1}{3} \mathrm{i} a_{n}^{2}\left(\frac{\sigma}{a_{n}^{2}}+\frac{9 K_{m n}-K_{n n}}{24 \omega_{m}}\right)$.

Consequently, the uncoupled mode is always marginally stable except when $\hat{\sigma}=\sigma / a_{n}^{2}=-\left(9 K_{m n}-\right.$ $\left.K_{n n}\right) /\left(24 \omega_{m}\right)$. At this level of detuning-to-squaredamplitude ratio, the two eigenvalues coalesce to zero and the uncoupled mode becomes degenerate.

On the other hand, for the coupled modes $a_{m} \neq 0$ and $a_{n} \neq 0$, we can solve for $\dot{\beta}_{m}$ and $\dot{\beta}_{n}$ and substitute the result into $\dot{\gamma}=\sigma-3 \dot{\beta}_{m}+\beta_{n}$ thereby yielding

$$
\begin{aligned}
\dot{\gamma}= & \sigma+\frac{1}{8 \omega_{m}}\left(3 K_{m m}-\frac{K_{m n}}{3}\right) a_{m}^{2} \\
& +\frac{1}{8 \omega_{m}}\left(3 K_{m n}-\frac{K_{n n}}{3}\right) a_{n}^{2} \\
& +\frac{\Lambda}{8 \omega_{m}} \cos \gamma\left(3 \frac{a_{m}}{a_{n}}-\frac{1}{9} \frac{a_{m}^{3}}{a_{n}^{3}}\right) a_{n}^{2} .
\end{aligned}
$$

Therefore, (129), (131), and (133) govern the amplitude- and phase-modulations of the coupled non-linear normal modes. Letting $c=a_{m} / a_{n}$ and assuming $\Lambda \neq 0$, the fixed points of these equations are given by

$$
\begin{gathered}
\alpha_{3} c^{3}+\alpha_{2} c^{2}+\alpha_{1} c+\alpha_{0}=0, \\
\gamma=n \pi, \quad n=0,1,2, \ldots
\end{gathered}
$$

where

$$
\begin{aligned}
& \alpha_{3}=\Lambda \cos \gamma, \quad \alpha_{2}=3\left(K_{m n}-9 K_{m m}\right), \quad \alpha_{1}=-27 \alpha_{3}, \\
& \alpha_{0}=3\left(K_{n n}-9 K_{m n}\right)-72 \omega_{m} \hat{\sigma} .
\end{aligned}
$$


(i)

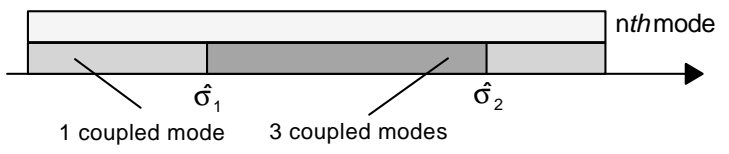

(ii)

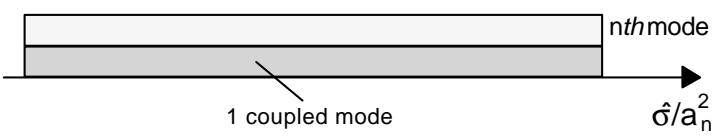

Fig. 2. Regions of existence of the non-linear normal modes due to a $3: 1$ internal resonance when (i) $\Delta>0$ and (ii) $\Delta<0$.

Because (134) is a cubic equation with real coefficients, it admits one or three real roots. Changing the phase by $\pi$ (i.e., $\gamma \rightarrow \gamma+\pi$ ) the coefficients of the odd powers of $c$ change sign; hence, the solutions experience a sign change (this property holds for a polynomial equation of arbitrary order). However, the overall solution - coupled mode - is invariant under the transformation $\gamma \rightarrow \gamma+\pi$ and $c \rightarrow-c$. Therefore, we can consider only the solutions associated with the phase $\gamma=2 n \pi$.

Consequently, the system possesses either two (one uncoupled and one coupled) or four (one uncoupled and three coupled) non-linear normal modes (Fig. 2). Clearly, local orthogonality never occurs also for three-to-one internal resonance.

The multivalued-range (three coupled non-linear normal modes) is $\hat{\sigma}_{1}<\hat{\sigma}<\hat{\sigma}_{2}$ when $\Delta>0$, where

$$
\begin{aligned}
\hat{\sigma}_{1,2}= & (g \mp 2 \sqrt{\Delta}) /\left(72 \Lambda^{2} \omega_{m}\right), \\
g= & -270 \Lambda^{2} K_{m m}-1458 K_{m m}^{3}+27 \Lambda^{2} K_{m n} \\
& +486 K_{m m}^{2} K_{m n}-54 K_{m m} K_{m n}^{2} \\
& +2 K_{m n}^{3}+3 \Lambda^{2} K_{n n}, \\
\Delta= & \left(9 \Lambda^{2}+81 K_{m m}^{2}-18 K_{m m} K_{m n}+K_{m n}^{2}\right)^{3} .
\end{aligned}
$$

On the other hand, when $\Delta<0$, there is only one coupled non-linear normal mode. This holds for $\gamma=n \pi$ with arbitrary integer $n$.

The stability of these modes can be determined by investigating the stability of the corresponding fixed points of (129), (131), and (133). One of the eigenvalues is zero and the other two are given by

$\lambda_{1,2}= \pm 72 \frac{a_{m} a_{n}}{\omega_{m}} \sqrt{\Delta_{\lambda}}$,

where

$$
\begin{aligned}
\Delta_{\lambda}= & \Lambda^{2}\left[-c^{4}-54 c^{2}+\frac{\cos \gamma}{\Lambda}\right. \\
& \left.\times\left(432 K_{m m}-54 K_{m n}+6 K_{n n}\right) c+243\right] .
\end{aligned}
$$

Evidently, the modes are marginally stable (centers) when $\Delta_{\lambda}<0$; are unstable (saddles) when $\Delta_{\lambda}>0$; and are degenerate when $\Delta_{\lambda}=0$ as they undergo a saddle-center bifurcation as documented in [10]. We note that also $\Delta_{\lambda}$ is invariant under the transformation $c \rightarrow-c$ and $\gamma \rightarrow \gamma+\pi$.

The displacement field, to second order, incorporating the solutions for $\beta_{m}$ and $\beta_{n}$, can be expressed as $u(x, t)$

$$
\begin{aligned}
= & a_{m} \cos \left(\omega_{m N} t+\beta_{m 0}\right) \phi_{m}(x) \\
& +a_{n} \cos \left[3\left(\omega_{m N} t+\beta_{m 0}\right)+\gamma\right] \phi_{n}(x) \\
& +\frac{1}{2}\left\{a_{m}^{2}\left[\cos 2\left(\omega_{m N} t+\beta_{m 0}\right) \Psi_{m m}(x)+\chi_{m m}(x)\right]\right. \\
& +a_{n}^{2}\left[\cos \left[6\left(\omega_{m N} t+\beta_{m 0}\right)+2 \gamma\right] \Psi_{n n}(x)+\chi_{n n}(x)\right] \\
& +a_{n} a_{m}\left[\cos \left[4\left(\omega_{m N} t+\beta_{m 0}\right)+\gamma\right] \Psi_{m n}(x)\right. \\
& \left.\left.+\cos \left[2\left(\omega_{m N} t+\beta_{m 0}\right)+\gamma\right] \chi_{m n}(x)\right]\right\},
\end{aligned}
$$

where

$$
\begin{aligned}
\omega_{m N}= & \omega_{m}-\left[\frac{\Lambda}{8 \omega_{m}} \cos \gamma a_{m} a_{n}\right] \\
& -\frac{K_{m m}}{8 \omega_{m}} a_{m}^{2}-\frac{K_{m n}}{8 \omega_{m}} a_{n}^{2}
\end{aligned}
$$

is the non-linear frequency expansion of the $m$ th mode.

Next, we discuss the condition for the global orthogonality of the normal modes.

Proposition IIa. A necessary and sufficient condition for orthogonality of the non-linear normal modes due to a three-to-one internal resonance is $\Lambda=0$.

Using the same line of proof employed for the two-to-one internal resonance, accounting for the expression (61) of $V^{(\sigma)}$ associated with this interaction, it can be easily ascertained that the proposition holds true. 
Also for this interaction, an interesting mechanical interpretation of the obtained non-linear orthogonality condition can be pursued. In fact, the terms $G_{2}\left(\phi_{k}, \Psi_{k k}\right)$ and $G_{2}\left(\Psi_{k k}, \phi_{k}\right)$ are the third-order effects of the quadratic elastic forces associated with twice the frequency of the $k$ th mode. On the other hand, the term $G_{3}\left(\phi_{k}, \phi_{k}, \phi_{k}\right)$ represents the cubic elastic force associated with the $k$ th mode. Therefore, the non-linear interaction coefficient $\Lambda_{n}=\Lambda / 3$ (see (109)) can be interpreted as the virtual work performed by the total third-order elastic forces associated with the low-frequency mode- $G_{2}\left(\phi_{m}, \Psi_{m m}\right)+$ $G_{2}\left(\Psi_{m m}, \phi_{m}\right)+G_{3}\left(\phi_{m}, \phi_{m}, \phi_{m}\right)$-in the displacement of the high-frequency mode. Therefore,

Proposition IIb. A necessary and sufficient condition for orthogonality of the non-linear normal modes due to a three-to-one internal resonance is that the virtual work performed by the total third-order low-frequency elastic forces in the displacement of the high-frequency mode be zero.

The non-linear interaction coefficient obtained with the discretization approach, (60), is a spectral representation of the above-mentioned virtual work in the eigenmode space. In particular, the part of it associated with the cubic forces is the same. The component associated with the third-order effect of the quadratic forces captures, in principle, contributions from all of the modes of the system. To conclude this section, it is worth noting the significant computational cost saved when ascertaining the non-linear orthogonality as illustrated here in contrast with performing the perturbation expansion up to third order. To check for non-linear orthogonality, it is simply required to compute: (i) the boundary-value problem yielding $\Psi_{m m}$ and (ii) the integrals in $\Lambda_{n}$.

\subsection{One-to-one internal resonances}

Separating real and imaginary parts in the modulation equations (65) and (66) yields their real-valued form as

$$
\begin{aligned}
\dot{a}_{m}= & \left(\frac{K_{1}}{8 \omega_{m}} a_{n}^{3}+\frac{K_{2}}{8 \omega_{m}} a_{m}^{2} a_{n}\right. \\
& \left.+\frac{K_{3}}{2 \omega_{m}} a_{m} a_{n}^{2} \cos \gamma\right) \sin \gamma,
\end{aligned}
$$

$$
\begin{aligned}
a_{m} \dot{\beta}_{m}= & -\frac{K_{m m}}{8 \omega_{m}} a_{m}^{3}-\frac{K_{m n}}{8 \omega_{m}} a_{m} a_{n}^{2}-\frac{K_{1}}{8 \omega_{m}} a_{n}^{3} \cos \gamma \\
& -\frac{3 K_{2}}{8 \omega_{m}} a_{m}^{2} a_{n} \cos \gamma-\frac{K_{3}}{4 \omega_{m}} a_{n}^{2} a_{m} \cos 2 \gamma, \\
\dot{a}_{n}=- & \left(\frac{K_{2}}{8 \omega_{n}} a_{m}^{3}+\frac{K_{1}}{8 \omega_{n}} a_{m} a_{n}^{2}\right. \\
+ & \left.\frac{K_{3}}{2 \omega_{n}} a_{m}^{2} a_{n} \cos \gamma\right) \sin \gamma, \\
\dot{a}_{n}= & -\frac{K_{n n}}{8 \omega_{n}} a_{n}^{3}-\frac{K_{m n}}{8 \omega_{n}} a_{n} a_{m}^{2}-\frac{K_{2}}{8 \omega_{n}} a_{m}^{3} \cos \gamma \\
& -\frac{3 K_{1}}{8 \omega_{n}} a_{m} a_{n}^{2} \cos \gamma-\frac{K_{3}}{4 \omega_{n}} a_{m}^{2} a_{n} \cos 2 \gamma,
\end{aligned}
$$

where $\gamma=\sigma t+\beta_{n}-\beta_{m}$.

When either both $K_{1} \neq 0$ and $K_{2} \neq 0$ or, simultaneously, $K_{1} \neq 0, K_{2} \neq 0$, and $K_{3} \neq 0$, the system possesses only coupled modes; that is, $a_{m} \neq 0$ and $a_{n} \neq 0$. In this case, solving for $\dot{\beta}_{m}$ and $\dot{\beta}_{n}$ and substituting into the phase equation yields

$$
\begin{aligned}
\dot{\gamma}= & \sigma+\frac{1}{8 \omega_{m}}\left(K_{m m}-K_{m n}-2 K_{3} \cos 2 \gamma\right) a_{m}^{2} \\
& +\frac{1}{8 \omega_{m}}\left(K_{m n}-K_{n n}+2 K_{3} \cos 2 \gamma\right) a_{n}^{2} \\
& +\frac{\cos \gamma}{8 \omega_{m}}\left[3\left(K_{2}-K_{1}\right) \frac{a_{m}}{a_{n}}+K_{1} \frac{a_{n}}{a_{m}}-K_{2} \frac{a_{m}^{3}}{a_{n}^{3}}\right] a_{n}^{2} .
\end{aligned}
$$

Inspecting (138) and (140), we conclude that there are two possible sets of coupled solutions: (a) $\sin \gamma=0$ or $\gamma=n \pi, n=0,1,2, \ldots$ and (b) $\sin \gamma \neq 0$. In case (a), the fixed points are solutions of the following equation:

$a_{4} c^{4}+a_{3} c^{3}+a_{2} c^{2}+a_{1} c+a_{0}=0$,

where

$$
\begin{aligned}
& a_{4}=K_{2} \cos \gamma, \quad a_{3}=\left(K_{m n}-K_{m m}+2 K_{3}\right), \\
& a_{2}=3\left(K_{1}-K_{2}\right) \cos \gamma, \\
& a_{1}=-\left(K_{m n}-K_{n n}+2 K_{3}\right)-8 \omega_{m} \hat{\sigma}, a_{0}=-K_{1} \cos \gamma,
\end{aligned}
$$

where $\hat{\sigma}=\sigma / a_{n}^{2}, \cos \gamma= \pm 1$ and use of the fact that $\cos 2 \gamma=1$ has been made.

Because (143) is a quartic equation with real coefficients, it possesses either four real solutions, or two 
real and two complex conjugate, or two pairs of complex conjugate solutions corresponding to $\cos \gamma=1$ and $\cos \gamma=-1$. Also in this case, the roots are subject to a sign change under the transformation $\gamma \rightarrow \gamma+\pi$ and they correspond to the same coupled modes. In this case, the coefficients of the even powers of $c$ change sign when the relative phase is shifted by $\pi$. Hence, there may be either four or two coupled non-linear normal modes corresponding to $\sin \gamma=0(\gamma=n \pi)$.

In case $(b)(\sin \gamma \neq 0)$, the coupled modes are solutions of

$b_{2} c^{2}-b_{1}+8 \omega_{m} \hat{\sigma}=0$,

where

$b_{2}=\frac{\left(K_{1} K_{2}-K_{2}^{2}\right)}{2 K_{3}}+K_{m m}-K_{m n}+2 K_{3}$,

$b_{1}=\frac{\left(K_{1} K_{2}-K_{1}^{2}\right)}{2 K_{3}}+K_{n n}-K_{m n}+2 K_{3}$.

The relative phase is given by

$\gamma=\cos ^{-1}\left[-\frac{K_{2} c^{2}+K_{1}}{4 K_{3} c}\right]+2 n \pi$.

The quadratic equation (144) admits two real solutions:

$c= \pm\left(\frac{b_{1}-8 \omega_{m} \hat{\sigma}}{b_{2}}\right)^{1 / 2}$

provided that $b_{2} \neq 0$ and the argument of the square root is positive. Therefore, considering the coupled normal modes corresponding to cases (a) and (b), we may have either six, four or two coupled non-linear normal modes. Discussion of the stability of the coupled modes in closed form is not a trivial task for this resonance condition and is not pursued here.

Instead, we analyze a simple case which often occurs in symmetric structural systems with a symmetric initial non-linear static configuration. As it is shown in Part II, for these systems, one-to-one internal resonances often occur due to crossovers between symmetric and antisymmetric modes. The result is that $K_{1}$ and $K_{2}$ are identically zero (in this case, the system would possess uncoupled modes as well, herein not considered); however, $K_{3} \neq 0$. Therefore, the coupled modes of cases (a) and (b) are given, respectively,

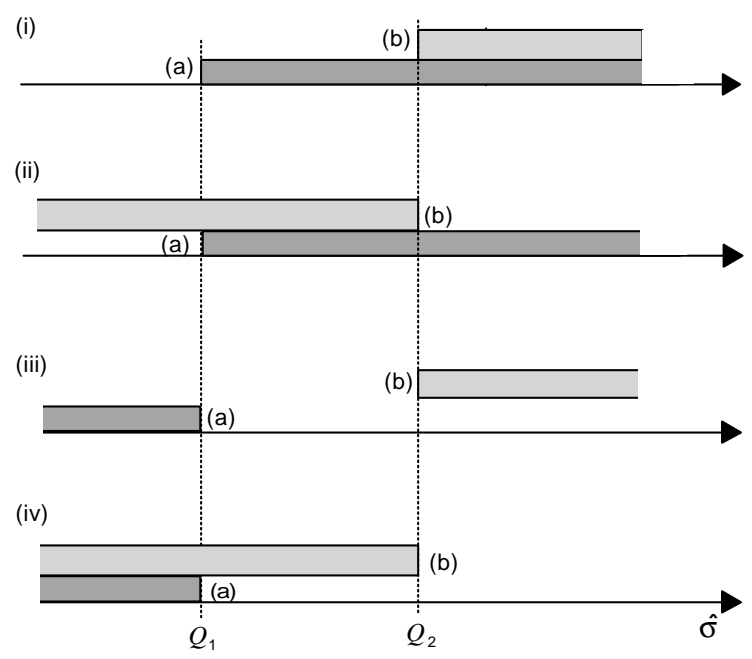

Fig. 3. Regions of existence of the non-linear normal modes of type (a) and (b) due to a 1:1 internal resonance when $K_{1}=K_{2}=0$ and (i) $P_{1}, P_{2}>0$, (ii) $P_{2}>0$ and $P_{1}<0$, (iii) $P_{2}<0$ and $P_{1}>0$, and (iv) $P_{1}, P_{2}<0$.

by

$$
\begin{aligned}
\text { (a) } c & = \pm\left(\frac{8 \omega_{m} \hat{\sigma}+K_{m n}-K_{n n}+2 K_{3}}{K_{m n}-K_{m m}+2 K_{3}}\right)^{1 / 2}, \\
\gamma & =n \pi,
\end{aligned}
$$

(b): $c= \pm\left(\frac{8 \omega_{m} \hat{\sigma}+K_{m n}-K_{n n}-2 K_{3}}{K_{m n}-K_{m m}-2 K_{3}}\right)^{1 / 2}$,

$$
\gamma=(2 n+1) \frac{\pi}{2} \text {. }
$$

In case (a), there are two real solutions when $\hat{\sigma} \gtrless Q_{1}$ if $P_{2} \gtrless 0$; in case (b), there are two real solutions when $\hat{\sigma} \gtrless Q_{2}$ if $P_{1} \gtrless 0$, where $Q_{1,2}=\left(K_{n n}-K_{m n} \mp\right.$ $\left.2 K_{3}\right) /\left(8 \omega_{m}\right)$ and $P_{1,2}=\left(K_{m n}-K_{m m} \mp 2 K_{3}\right)$. These ranges of existence of the normal modes are illustrated in Fig. 3.

For sake of discussion, let us assume that $P_{1}>0$ and $P_{2}<0$; then, the system possesses the coupled mode (a) when $\hat{\sigma}<Q_{1}$ and the coupled mode (b) when $\hat{\sigma}>Q_{2}$ (Fig. 3). Therefore, when $Q_{1}<\hat{\sigma}<Q_{2}$, there are no solutions; hence, there are no resonant coupled modes. This is a region of local uncoupling in the detuning-amplitude plane. In this region, the modes cannot interact in a periodic fashion. Similar regions occur in cases (i) and (iv) but not in case (ii) in Fig. 3. 
Coming back to the general case, the displacement field of the resonant normal modes can be expressed, to second order, as

$$
\begin{aligned}
u(x, t)= & a_{m} \cos \left(\omega_{m N} t+\beta_{m 0}\right) \phi_{m}(x) \\
& +a_{n} \cos \left[\left(\omega_{m N} t+\beta_{m 0}\right)+\gamma\right] \phi_{n}(x) \\
& +\frac{1}{2}\left\{a_{m}^{2}\left[\cos 2\left(\omega_{m N} t+\beta_{m 0}\right) \Psi_{m m}(x)+\chi_{m m}(x)\right]\right. \\
& +a_{n}^{2}\left[\cos 2\left[\left(\omega_{m N} t+\beta_{m 0}\right)+\gamma\right] \Psi_{n n}(x)+\chi_{n n}(x)\right] \\
& +a_{n} a_{m}\left[\cos \left[2\left(\omega_{m N} t+\beta_{m 0}\right)+\gamma\right] \Psi_{m n}(x)\right. \\
& \left.\left.+\cos \gamma \chi_{m n}(x)\right]\right\},
\end{aligned}
$$

where the non-linear frequency expansion of the $m$ th mode is given by

$$
\begin{aligned}
\omega_{m N}= & \omega_{m}-\left[\frac{K_{1}}{8 \omega_{m}} \frac{a_{n}^{3}}{a_{m}}+3 \frac{K_{2}}{8 \omega_{m}} a_{m} a_{n}+\frac{K_{3}}{2 \omega_{m}} a_{n}^{2} \sin \gamma\right] \\
& \times \cos \gamma-\frac{K_{m m}}{8 \omega_{m}} a_{m}^{2}-\frac{K_{m n}}{8 \omega_{m}} a_{n}^{2} .
\end{aligned}
$$

Next, the condition for the global orthogonality of the normal modes in the presence of a one-to-one internal resonance is discussed.

Proposition III. Necessary and sufficient conditions for orthogonality of the non-linear normal modes due to a one-to-one internal resonance are $K_{1}=0, K_{2}=0$, and $K_{3}=0$.

The arguments leading to this proposition are the same as those provided for two-to-one and three-to-one interactions. These non-linear orthogonality conditions do not lend themselves to straightforward mechanical interpretations as for the cases of two-to-one and three-to-one internal resonances. Here, they represent some more complex forms of virtual works (mixed virtual works). However, we emphasize the relatively low computational cost required for ascertaining the non-linear orthogonality. To this end, it is needed to compute (i) four boundary-value problems yielding $\Psi_{m m}, \Psi_{n n}, \chi_{m m}$, and $\chi_{m n}$, respectively, and (ii) the integrals expressing $K_{1}, K_{2}$, and $K_{3}($ see $(110)-(112))$.

\section{Conclusions}

Coupled/uncoupled resonant non-linear normal modes have been constructed in the cases of two-to-one, three-to-one, and one-to-one internal resonances. The method of multiple scales has been applied to the full-basis Galerkin-reduced model or directly to the equations of motion and boundary conditions of a general class of one-dimensional continuous systems. These systems are non-linear with weak quadratic and cubic non-linearities. The objective of this work is twofold: (i) to study the existence and stability of coupled/uncoupled non-linear normal modes using a general and systematic approach; (ii) to determine closed-form conditions for the non-linear global and local orthogonality of the modes.

Regarding the first issue, we determined the number of coupled/uncoupled non-linear normal modes and the associated stability in the detuning-amplitude space. In the case of a two-to-one internal resonance, it was also established that a higher-order approximation (i) does not affect the activation of the internal resonance; (ii) does not influence the relative phase of the interacting modes; and (iii) does affect the detuning-amplitude relationship of the modes and the associated stability bounds.

The normal modes obtained with the full-basis discretization approach are the same as those obtained with direct application of the method of multiple scales to the equations of motion and boundary conditions. Exploiting the equivalence of the results obtained with the two analytical treatments is important because the direct approach only yields closed-form expressions of the interaction coefficients in actual computations. In turn, the full-basis approach yields interesting modal representations of the coupled/uncoupled normal modes. These spectral representations, besides shedding light onto the structure of these non-linear modes, may be useful for modal convergence investigations and reduced-order models selection.

Necessary and sufficient conditions for non-linear orthogonality of the modes, in a local and global sense, have been determined and discussed. In the cases of two-to-one and three-to-one interactions, based on the results of the direct approach, these conditions can be interpreted as vanishing of pertinent virtual works thereby extending the mechanical concept of linear 
orthogonality to the weakly non-linear vibration regime of self-adjoint systems. The obtained orthogonality conditions may save significant computational costs. In fact, to ascertain the occurrence of global non-linear orthogonality between two modes, it is needed to: (i) compute some integrals involving the modes and their derivatives for two-to-one interactions; (ii) solve one differential boundary-value problem (yielding $\Psi_{m m}$ ) and perform some integrations to evaluate the virtual work defined for three-to-one interactions; (iii) solve four differential boundary-value problems (yielding $\Psi_{m m}, \Psi_{n n}, \chi_{m m}$, and $\chi_{m n}$ ) and evaluate a number of integrals leading to three independent interaction coefficients for one-to-one internal resonances.

These relatively simple computations (solutions of boundary-value problems and straightforward integrations) save the effort of constructing approximations to the sought normal modes using an asymptotic technique up to the resonant non-linear order. The presented closed-form conditions allow to conclude a priori on orthogonality or non-orthogonality of the modes. While the latter leads to non-linear modal interactions, the former, in its global form, prevents the internal resonance from being activated.

\section{Acknowledgements}

This work was partially supported by the M.U.R. S.T. grant on Non-linear Dynamics, Stability, and Control of Solids and Structures.

\section{References}

[1] G. Rega, R. Alaggio, F. Benedettini, Experimental investigation of the non-linear response of a hanging cable. Part I: local analysis, Nonlinear Dynamics 14 (1997) 89.

[2] A.H. Nayfeh, Non-linear Interactions, Wiley Interscience, New York, 2000.

[3] R.M. Rosenberg, The normal modes of nonlinear $N$-degree-of-freedom systems, J. Appl. Mech. 30 (1962) 7.

[4] M.E. King, A.F. Vakakis, An energy-based formulation for computing nonlinear normal modes in undamped continuous systems, J. Vibr. Acoustics 116 (1993) 332.

[5] M.E. King, A.F. Vakakis, An energy-based approach to computing resonant nonlinear normal modes, J. Appl. Mech. 63 (1996) 810.
[6] S.W. Shaw, C. Pierre, Normal modes of vibration for nonlinear continuous systems, J. Sound Vibr. 169 (1994) 319.

[7] N. Boivin, C. Pierre, S.W. Shaw, Non-linear modal analysis of structural systems featuring internal resonances, J. Sound Vibr. 182 (1995) 336.

[8] A.H. Nayfeh, On direct methods for constructing nonlinear normal modes of continuous systems, J. Vibr. Control 1 (1995) 389.

[9] A.H. Nayfeh, C.-M. Chin, S.A. Nayfeh, On nonlinear normal modes of systems with internal resonance, J. Vibr. Acoustics 118 (1996) 340.

[10] A.H. Nayfeh, W. Lacarbonara, C.-M. Chin, Nonlinear normal modes of buckled beams: Three-to-one and one-to-one internal resonances. Nonlinear Dynamics 18 (1999) 253.

[11] W. Lacarbonara, G. Rega, Resonant non-linear normal modes. Part II: activation/orthogonality conditions for shallow structural systems, Int. J. Non-Linear Mech. 37 (2002).

[12] A.H. Nayfeh, S.A. Nayfeh, M. Pakdemirli, On the discretization of weakly nonlinear spatially continuous systems, in: W. Kliemann, N. Sri Namachchivaya (Eds.), Nonlinear Dynamics and Stochastic Mechanics, CRC Press, Boca Raton, FL, 1995, p. 175.

[13] M. Pakdemirli, H. Boyaci, Vibrations of continuous systems having arbitrary quadratic and cubic nonlinearities, Appl. Mech. Eng. 1 (1996) 445.

[14] W. Lacarbonara, Direct treatment and discretizations of nonlinear spatially continuous systems, J. Sound Vibr. 221 (1999) 849.

[15] W.F. Simmons, A variational method for weak resonant wave interactions, Proc. Roy. Soc. A 309 (1969) 551.

[16] M. Pakdemirli, A comparison of two perturbation methods for vibrations of systems with quadratic and cubic nonlinearities, Mech. Res. Commun. 21 (1994) 203.

[17] A.H. Nayfeh, Reduced-order models of weakly nonlinear spatially continuous systems, Nonlinear Dynamics 16 (1998) 105.

[18] A.H. Nayfeh, A.H. Arafat, C.-M. Chin, W. Lacarbonara, Multimode interactions in suspended cables, J. Vibr. Control, 2002, in press.

[19] J.A. Murdock, Perturbations. Theory and Methods, Wiley-Interscience, New York, 1991.

[20] Z.C. Feng, L.G. Leal, Symmetries of the amplitude equations of an inextensional beam with internal resonance, J. Appl. Mech. 62 (1994) 235.

[21] H.M. Irvine, T.K. Caughey, The linear theory of free vibrations of a suspended cable, Proc. Roy. Soc. London, Ser. A 341 (1974) 299.

[22] A.W. Leissa, On a curve veering abberation, J. Appl. Math. Phys. (ZAMP) 25 (1974) 99.

[23] N.C. Perkins, C.D. Mote Jr., Comments on curve veering in eigenvalue problems, J. Sound Vibr. 106 (1986) 451.

[24] A.H. Nayfeh, B. Balachandran, Applied Nonlinear Dynamics, Wiley Interscience, New York, 1995. 